\title{
Sum-of-squares Flight Control Synthesis for Deep-stall Recovery
}

\author{
Torbjørn Cunis* \\ ONERA - The French Aerospace Lab, Centre Midi-Pyrénées, Toulouse, 31055, France \\ Jean-Philippe Condomines ${ }^{\dagger}$ \\ ENAC, Université de Toulouse, Toulouse, 31055, France \\ Laurent Burlion ${ }^{\ddagger}$ \\ Rutgers, The State University of New Jersey, Piscataway, NJ 08854, USA
}

Certification of inflight loss-of-control recovery is complicated by the highly nonlinear flight dynamics beyond stall. In lieu of extensive Monte-Carlo simulations for flight control certification, sum-of-squares programming techniques provide an algebraic approach to the problem of nonlinear control synthesis and analysis. However, reliance on polynomial models has hitherto limited applicability to aeronautical control problems. Taking advantage of recently proposed piecewise polynomial models, this paper revisits sum-of-squares techniques for recovery of an aircraft from deep-stall conditions using a realistic yet tractable aerodynamic model. Local stability analysis of classical controllers is presented as well as synthesis of polynomial feedback laws with the objective of enlarging their nonlinear region of attraction. A newly developed synthesis algorithm for infinite-horizon backwards-reachability facilitates the design of recovery control laws, ensuring stable recovery by design. The paper's results motivate future research in aeronautical sum-of-squares applications.

\section{Nomenclature}

$\alpha$

$\alpha_{0}$

$\gamma_{\mathrm{A}}$

$\eta$
Angle of attack (rad);

Low-angle of attack boundary $\left(\alpha_{0}=16.2949^{\circ}\right)$;

Flight-path angle relative to air (rad);

Elevator deflection (rad), negative if leading to positive pitch moment;

\footnotetext{
${ }^{*}$ Now with the University of Michigan, Ann Arbor, Michigan 48109, USA, e-mail: tcunis@umich.edu Member AIAA.

$\dagger$ Assistant Professor, Drones Research Group, e-mail: jean-philippe.condomines@enac.fr

$\ddagger$ Assistant Professor, Department of Mechanical \& Aerospace Engineering, e-mail: laurent.burlion@rutgers.edu
} 


\begin{tabular}{|c|c|}
\hline$\vartheta$ & Signed distance ratio $(\vartheta \in \mathbb{R})$; \\
\hline$\lambda$ & Level set $\left(\lambda \in \mathbb{R}_{+}\right)$; \\
\hline$\rho$ & Pseudo-radius $\left(\rho \in \mathbb{R}_{+}\right)$; \\
\hline$\varphi(\cdot)$ & Boundary condition function $\left(\varphi: \mathbb{R}^{m} \rightarrow \mathbb{R}\right)$ \\
\hline$d \eta$ & Change of elevator deflection $(\mathrm{rad} / \mathrm{s})$; \\
\hline $\mathbf{K}$ & Feedback control law $\left(\mathbf{K}: \mathbb{R}^{n} \rightarrow \mathbb{R}^{p}\right)$ \\
\hline$n$ & Number of states; system degree; \\
\hline$p$ & Number of inputs; \\
\hline$P$ & Positive-definite, polynomial shape function $\left(P: \mathbb{R}^{n} \rightarrow \mathbb{R}_{\geq 0}, P \in \mathbb{R}[\mathbf{x}]\right)$ \\
\hline$q$ & Pitch rate $(\mathrm{rad} / \mathrm{s})$ \\
\hline$V_{\mathrm{A}}$ & Aircraft speed relative to air $(\mathrm{m} / \mathrm{s})$, positive along $x_{\mathrm{a}}$-axis; \\
\hline $\mathbf{x}^{*}, \eta^{*}$ & State vector and elevator deflection at trim condition; \\
\hline$\tilde{\mathbf{x}}, \tilde{\eta}$ & Scaled state vector and elevator deflection; \\
\hline$(\cdot)^{p o s t}$ & Domain of high angle of attack; \\
\hline$(\cdot)^{p r e}$ & Domain of low angle of attack; \\
\hline $\mathcal{E}$ & (Quasi)-Ellipsoidal set $(\mathcal{E}=\{\mathbf{x} \mid P(\mathbf{x}) \leq \rho\})$ with shape function $P$ and pseudo-radius $\rho$; \\
\hline$\Sigma[\mathbf{x}]$ & Polynomial sum-of-squares cone $(\Sigma[\mathbf{x}] \subset \mathbb{R}[\mathbf{x}])$; \\
\hline $\mathbb{R}[\mathbf{x}]$ & Set of polynomials in $\mathbf{x}$ with real-valued coefficients; \\
\hline
\end{tabular}

\section{Introduction}

Pediction and PREvention of inflight loss-of-control (LOC-I) commonly requires prior knowledge 1 of the aircraft's dynamics, often given by a reliable and representative aerodynamic model. However, establishing a satisfactory model is rarely straightforward and ensuring sufficient representation of every aspect of the operational envelope of the true vehicle is extremely challenging. Indeed, dynamics beyond the nominal flight envelope are highly nonlinear and often unstable. Flight control certification for commercial airliners therefore relies today on simple but extensive Monte-Carlo simulations of high-fidelity models [1] in 
order to analyse the viable subset of the flight envelope; this demands significant investment of time and computational power. Yet, more sophisticated tools based on nonlinear stability theory have been applied in the literature. Mathematical continuation and bifurcation analysis establishes trim conditions and periodic orbits as well as their stability [2, 3]; however, attraction or reachability of a stable solution is not determined quantitatively by the continuation methodology. Reachability analysis, on the other hand, numerically evolves reachable subsets of the state-space over time, identifying possible violations of predefined constraints [4, 5]. An alternative formulation of the reachability problem is the algebraic notion of controlled invariant sets, or safe sets [6, 7]. Defined as the largest set such that the aircraft can be kept within the state constraints subject to control input limitations, the safe set determines bounds for prevention and recoverability from LOC-I events. The idea of a safe set as defined by the existence of an admissible control sequences is thus contrasted by the set of converging state trajectories subject to an a priori specified control law, namely, the region of attraction of the closed-loop system.

Determining the region of attraction of a given system up to a desired accuracy is, in general, a non-trivial task [8. Recently, Lyapunov stability theory and LaSalle's later extension have been turned into a systematic analysis approach employing sum-of-squares (SOS) programming techniques [9, 10]. Relaxed to semi-definite problems [11], SOS provides global stability proofs [12] as well as provable under-estimates for the region of attraction of systems defined by polynomial dynamics [13]. Those methods for stability analysis can be further extended to synthesis of control feedback laws ensuring or enlarging a region of attraction subject to input constraints [14, 15].

Sum-of-squares techniques have been exploited to analyze the short-period motion of an F/A-18 aircraft model [16. A special iteration technique, called $\mathcal{V}$-s-iteration, was applied to estimate the region of attraction of the longitudinal motion of the Generic Transport Model [17]. In [18, this technique was employed to validate a revised control law for the F/A-18 falling-leaf mode. Note that this work used a reduced six-state polynomial aircraft model of 3rd order, which was derived by sampling the equations of motion rather than the aerodynamic coefficients. However, this model assumes a constant airspeed. Despite SOS techniques being a powerful tool to generate Lyapunov functions for suitable models, few works on SOS for aircraft dynamics have been published since. Mere polynomials are often unsuitable to fit full-envelope aerodynamics accurately, whereas advanced modeling techniques, such as multivariate splines [19], are too computationally heavy to analyze using SOS. Simple piecewise-defined models, as proposed by the authors in [20], have the potential of bridging this gap; namely, these models accurately describe aircraft dynamics in the domains of low and high angles of attack while only slightly increasing the computational load for sum-of-squares programming.

In this article, we synthesize controllers for and verify deep-stall recovery of a small unmanned aircraft 
using SOS programming and a piecewise, 5th-order polynomial model. The choice of aircraft, a fixed-wing capable of stable deep-stall transition, descent, and recovery [21], allows us to isolate longitudinal dynamics. Continuing our work in [20], we present an extended $\mathcal{V}$-s-iteration for piecewise-defined aircraft dynamics in order to obtain feedback control laws subject to state and inputs constraints. In particular, we focus on two aspects: Analysis of an aircraft model with separately modelled, high angle-of-attack dynamics; and the synthesis of a control feedback to specifically recover from a deep-stall trim condition. While we initially rely on common polynomial surfaces to govern the estimate (cf. [13, 14, 17, 18]), we later replace it by a single deep-stall reachability condition. We thus provide a systematic analysis by SOS beyond polynomial aircraft models.

The paper is organized as follows: Section II introduces the aircraft, its piecewise polynomial model and recalls the basics of SOS analysis. Section III analyzes stable recovery of a linear-quadratic regulator and synthesizes polynomial controllers subject to an enlarged region of attraction. Section IV]concludes the article with a revised formulation of control synthesis specifically for deep-stall recovery.

Note on polynomial surfaces For sum-of-squares analysis and control synthesis, we make use of geometric objects defined by polynomial functions on the state-space, i.e., $\mathcal{E}=\{\mathbf{x} \mid, P(\mathbf{x}) \leq \rho\}$, where $P$ is a polynomial in $\mathbf{x}$ and $\rho>0$ determines the size of $\mathcal{E}$. The most common examples are ellipsoids (in three dimensions) and the related hyper-ellipsoidal surfaces, which are governed by quadratic functions $P=\mathbf{x}^{T} \mathbf{Q} \mathbf{x}$ with positive-definite matrix Q. For simplicity, we call both ellipsoids and hyper-ellipsoids ellipsoidal surfaces. The concept of (hyper)-ellipsoids can be further extended to positive polynomial surfaces of order larger than two, which we will refer to as quasi-ellipsoids.

\section{Methodology}

We consider an autonomous, 1.65 m-wingspan, unmanned aircraft that, instead of landing gear, is intended to land vertically by descending using a deliberate deep-stall maneuver. In this maneuver, the drag of the wings counteract the gravitational force, leading to a stable trim condition, and the horizontal distance covered during the landing is minimized. For the purpose of stable deep-stall flight, the elevator is designed to exceed the usual range and reach deflections of up to $-60^{\circ}$. The parameters of the aircraft used in this study are given by Tab. 1 .

The aircraft is considered to be laterally stable due to its dihedral wings. We therefore neglect the lateral dynamics for the analysis of stability and, consequently, assume that the side-slip angle $\beta$ vanishes. In deep-stall flight and transition, the aircraft is further unthrottled, i.e., the thrust force is zero $(\mathbf{F}=0)$.

We will refer mainly to the international standard air-path axis system $\left(x_{\mathrm{a}}, y_{\mathrm{a}}, z_{\mathrm{a}}\right)$ oriented along the 
Table 1 Parameters of the aircraft.

\begin{tabular}{rcll}
\hline flight mass & $m$ & 1.55 & $\mathrm{~kg}$ \\
wing span & $b$ & 1.66 & $\mathrm{~m}$ \\
mean chord & $c_{\mathrm{a}}$ & 0.174 & $\mathrm{~m}$ \\
wing area & $S$ & 0.277 & $\mathrm{~m}^{2}$ \\
\hline air density & $\varrho$ & 1.25 & $\mathrm{~kg} / \mathrm{m}^{3}$ \\
gravitational constant & $g$ & 9.81 & $\mathrm{~m} / \mathrm{s}^{2}$ \\
\hline
\end{tabular}

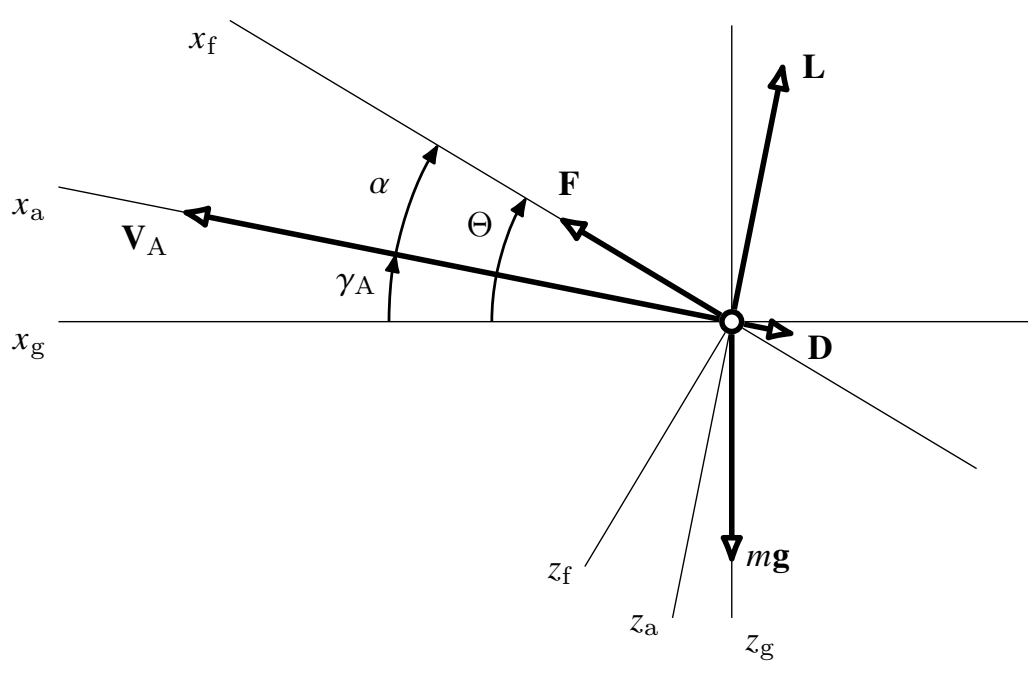

Fig. 1 Longitudinal axes with angles and vectors for $\beta=0$.

aircraft's velocity vector with respect to air $\left(\mathbf{V}_{\mathrm{A}}\right)$ 22]. Lift and drag forces are defined along these axes and denoted $\mathbf{L}$ and $\mathbf{D}$; angle of attack $\alpha$, flight-path angle $\gamma_{\mathrm{A}}$, and pitch angle $\Theta$ are given by rotations into the body axis system $\left(x_{\mathrm{f}}, y_{\mathrm{f}}, z_{\mathrm{f}}\right)$ as well as the earth-fixed axis system $\left(x_{\mathrm{g}}, y_{\mathrm{g}}, z_{\mathrm{g}}\right)$, defined by the aircraft's fuselage and the ground. (Fig. 1). If not stated otherwise, all variables are in SI-units; angles are however given in degrees where convenient.

A polynomial $g \in \mathbb{R}[\mathbf{x}]$ is a sum of squares (SOS) if $g=\sum_{i} g_{i}(\mathbf{x})^{2}$ for some $\left(g_{i}\right)_{i} \subset \mathbb{R}[\mathbf{x}]$; the set of sum-of-squares polynomials is denoted by $\Sigma[\mathbf{x}]$. It can be proven that $g \in \Sigma[\mathbf{x}]$ if and only if there is a positive semidefinite matrix $\mathbf{M}$ such that $g=\mathbf{z}^{T} \mathbf{M z}$, where $\mathbf{z}$ is a vector of monomials in $\mathbf{x}$ [10. This relation reduces the problem of finding SOS polynomials to semidefinite programming [11, given that the objective is linear in the SOS variables. Bilinear problems are generally $\mathcal{N} \mathcal{P}$-hard; however, the problem of a single, scalar decision variable entering bilinearly into the objective can be efficiently solved as a quasi-convex SOS program [10. Notwithstanding that any SOS polynomial is nonnegative, the opposite does not hold. 


\section{A. Equations of motion}

The longitudinal equations of motion without thrust are given as nonlinear 4-state, ordinary differential equations [17, 23, 24]:

$$
\begin{aligned}
\dot{V}_{\mathrm{A}} & =-\frac{1}{2} \varrho V_{\mathrm{A}}^{2} S m^{-1} C_{\mathrm{D}}(\alpha, \eta)-g \sin \gamma_{\mathrm{A}} \\
V_{\mathrm{A}} \dot{\gamma}_{\mathrm{A}} & =\frac{1}{2} \varrho V_{\mathrm{A}}^{2} S m^{-1} C_{\mathrm{L}}(\alpha, \eta)-g \cos \gamma_{\mathrm{A}} \\
I_{y} \dot{q} & =\frac{1}{2} \varrho V_{\mathrm{A}}^{2} S c_{\mathrm{a}} C_{\mathrm{m}}(\alpha, \eta)-k_{\dot{q} q} q \\
\dot{\Theta} & =q
\end{aligned}
$$

with the pitch angle

$$
\Theta=\gamma_{\mathrm{A}}+\alpha
$$

and the air speed $V_{\mathrm{A}}$ equal to the norm of the velocity relative to air. $C_{\mathrm{L}}, C_{\mathrm{D}}, C_{\mathrm{m}}$ are dimensionless coefficients connected to lift force, drag force, and pitching moment. The elevator deflection $\eta$ is, by convention, negative when causing a positive pitching moment. The linear damping coefficient $k_{\dot{q} q}$ accounts for non-static aerodynamics (see [21]).

The aerodynamic coefficients of the body, wing, and surfaces have been modeled by piecewise polynomial functions

$$
C_{\odot}(\alpha, \cdots)=\left\{\begin{array}{cc}
C_{\odot}^{\text {pre }}(\alpha, \cdots) & \text { if } \alpha \leq \alpha_{0} \\
C_{\odot}^{\text {post }}(\alpha, \cdots) & \text { otherwise }
\end{array}\right.
$$

with $C_{\odot}^{\text {pre }}\left(\alpha_{0}, \cdot\right) \equiv C_{\odot}^{\text {post }}\left(\alpha_{0}, \cdot\right)$ and $\alpha_{0}=16.2949^{\circ}$. Fig. 2 shows the piecewise model and their polynomial segments. Defined as piecewise polynomials, we are able to account for full-envelope characteristics both of the lift and drag coefficients as well as the coefficients in body axes [20. The resulting models are continuous over the entire domain but not necessarily differentiable in its joint. The pitch-moment coefficient $C_{\mathrm{m}}$ is modeled likewise. The full aircraft model is detailed in [25].

\section{B. Region of attraction estimation}

In the following, we use SOS programming to compute a region of attraction estimate for a piecewise polynomial system under constrained control inputs. We then extend this framework to find a control law that enlarges the region of attraction of the controlled system. In order to resolve the resulting bilinear terms, 


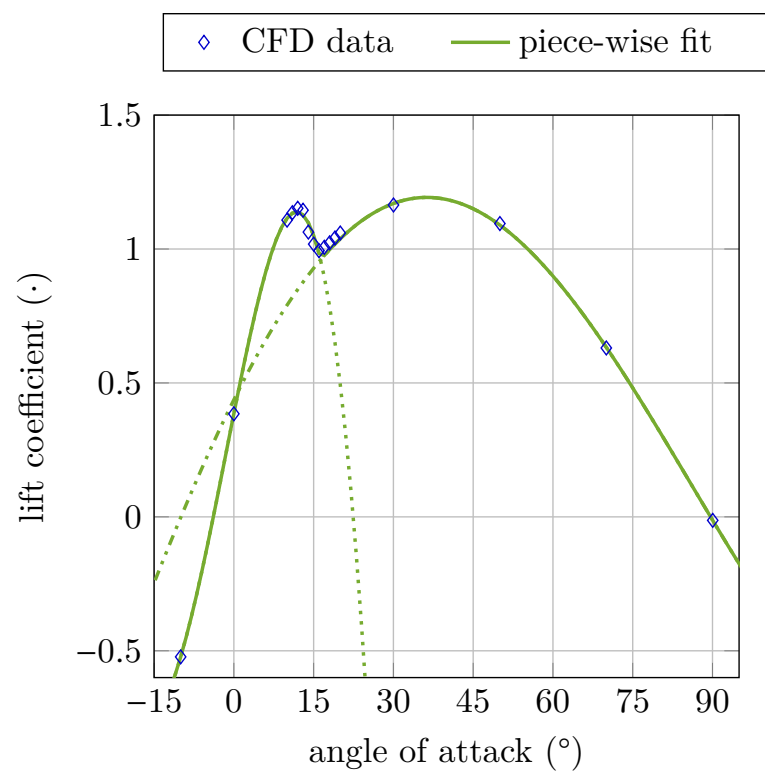

(a) Aerodynamic $C_{\mathrm{L}}$ coefficient.

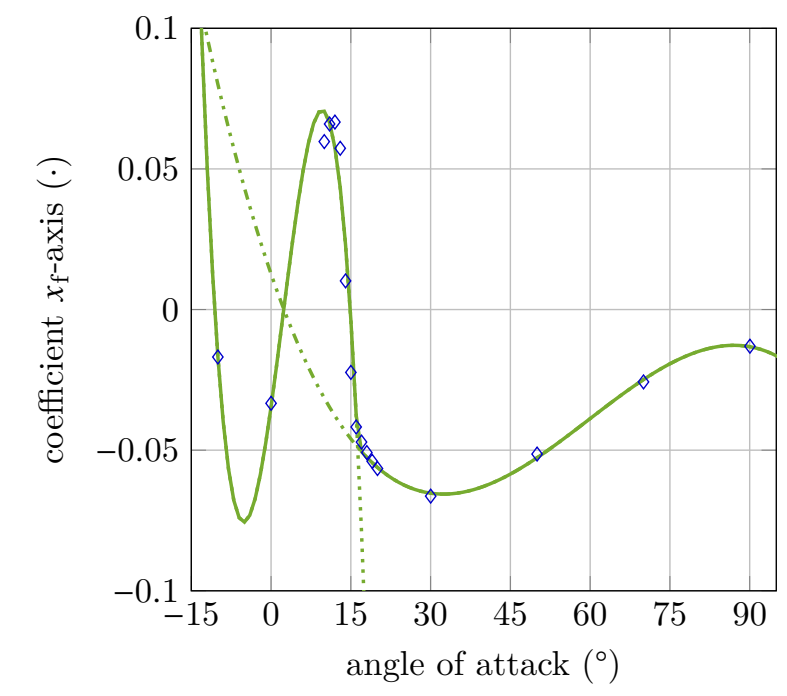

(b) Aerodynamic $C_{\mathrm{X}}$ coefficient.

Fig. 2 Piecewise model of aerodynamic coefficients with polynomial segments [21].

we will make use of the $\mathcal{V}$-s-iteration [17].

Let a piecewise polynomial system be defined as

$$
\mathbf{f}(\mathbf{x}, \mathbf{u})= \begin{cases}\mathbf{f}_{1}(\mathbf{x}, \mathbf{u}) & \text { if } \varphi(\mathbf{x}) \leq 0 \\ \mathbf{f}_{2}(\mathbf{x}, \mathbf{u}) & \text { otherwise }\end{cases}
$$

with state vector $\mathbf{x} \in \mathcal{X} \subset \mathbb{R}^{n}$, input vector $\mathbf{u} \in \mathcal{U} \subset \mathbb{R}^{p}$, submodels $\mathbf{f}_{1}, \mathbf{f}_{2} \in \mathbb{R}[\mathbf{x}, \mathbf{u}]^{n}$ and boundary $\varphi \in \mathbb{R}[\mathbf{x}]$; assume further $\mathbf{f}\left(\mathbf{x}^{*}, \mathbf{u}^{*}\right)=0$. The equilibrium $\left(\mathbf{x}^{*}, \mathbf{u}^{*}\right)$ is asymptotically stable if there exists a non-empty set of initial conditions $\mathcal{R}$, the region of attraction, such that the trajectories converge to $\mathbf{x}^{*}$ and are contained in $\mathcal{R}$, and $\mathcal{R}$ contains $\mathbf{x}^{*}$ in its interior. Now, $\Omega_{\lambda}=\{\mathbf{x} \mid \mathcal{V}(\mathbf{x}) \leq \lambda\}$ is an invariant subset of $\mathcal{R}$ if, for $\mathcal{V}: \mathcal{X} \rightarrow \mathbb{R}$ continuous and positive definite, $\mathcal{V}(0)=0$, and $\lambda \in \mathbb{R}_{+}$,

$$
\forall \mathbf{x} \in \Omega_{\lambda}, \quad \nabla \mathcal{V} \mathbf{f}\left(\mathbf{x}, \mathbf{u}^{*}\right)<0
$$

and $\Omega_{\lambda}$ is bounded [8]. Moreover, we call $\Omega_{\lambda}$ invariant under control $\mathbf{K}(\cdot)$ if $\Omega_{\lambda}$ is an invariant set of the closed-loop system $\mathbf{f}_{\mathbf{K}}: \mathbf{x} \mapsto \mathbf{f}(\mathbf{x}, \mathbf{K}(\mathbf{x}))$ for some control law $\mathbf{u}=\mathbf{K}(\mathbf{x})$ and $\mathbf{K}(\mathbf{x}) \in \mathcal{U}$ for all $\mathbf{x} \in \Omega_{\lambda}$.

As Lyapunov functions are non-unique, alternative $\mathcal{V}(\cdot)$ give rise to different estimates of the region of attraction. For comparison of the size of an invariant subset, the $\mathcal{V}$-s-iteration introduces a surface $\mathcal{E}_{\rho}=\{\mathbf{x} \mid P(\mathbf{x}) \leq \rho\}$ with $P \in \mathbb{R}[\mathbf{x}]$ positive (quadratic) chosen as parameter of the estimation [13]. The 
estimation of a maximal invariant subset of the region of attraction is then subject to the optimization problem

$$
\max _{\substack{\mathcal{V} \in \mathbb{R}[\mathbf{x}] \\ \lambda>0}} \rho>0 \quad \text { s.t. } \Omega_{\lambda} \subset\left\{x \mid \nabla \mathcal{V} \mathbf{f}\left(\mathbf{x}, \mathbf{u}^{*}\right)<0\right\} \text { and } \mathcal{E}_{\rho} \subset \Omega_{\lambda}
$$

as well as $\mathcal{V}(\mathbf{x})>0$ if $\mathbf{x} \neq 0$ and $\mathcal{V}(0)=0$.

\section{Piecewise region of attraction}

Recall that $\Omega_{\lambda}$ bounded is an invariant subset of $\mathcal{R}$ under control $\mathbf{K}$ if $\mathcal{V}(\cdot)$ is a continuous and positive definite function and $\nabla \mathcal{V} \mathbf{f}_{\mathbf{K} i}(x)<0$ for any $\mathbf{x} \in \Omega_{\lambda}$ and $i \in\{1,2\}$ such that $\mathbf{f}_{\mathbf{K} i}$ is active. For $\mathcal{V}_{1,2} \in \mathbb{R}[\mathbf{x}]$, we have the sufficient SOS constraint [20] (see also [13, Lemma 10])

$$
\begin{array}{r}
\mathcal{V}_{1,2}(\mathbf{x})-\ell_{a} \in \Sigma[\mathbf{x}] \\
-\nabla \mathcal{V}_{1} \mathbf{f}_{\mathbf{K} 1}-\ell_{b}+\left(\mathcal{V}_{1}-\lambda\right) s_{1, \lambda}+\varphi s_{1, \varphi} \in \Sigma[\mathbf{x}] \\
-\nabla \mathcal{V}_{2} \mathbf{f}_{\mathbf{K} 2}-\ell_{b}+\left(\mathcal{V}_{2}-\lambda\right) s_{2, \lambda}+\varphi s_{2, \varphi} \in \Sigma[\mathbf{x}]
\end{array}
$$

where $s_{i, \lambda}, s_{i, \varphi} \in \Sigma[\mathbf{x}], i \in\{1,2\}$ relax negativity of $\nabla \mathcal{V}_{i} \mathbf{f}_{i}$ to the respective partitions defined by $\Omega_{\lambda}$ and $\varphi(\cdot)$ and $\ell_{a, b} \in \mathbb{R}[\mathbf{x}]$ are positive definite terms [13], e.g., $\ell_{a}=\ell_{b}=\epsilon \mathbf{x}^{T} \mathbf{x}$ with some small $\epsilon>0$. As $\mathcal{V}(\cdot)$ is defined piecewise itself, i.e., $\mathcal{V}(\mathbf{x})=\mathcal{V}_{1}(\mathbf{x})$ if $\varphi(\mathbf{x}) \leq 0$; otherwise $\mathcal{V}(\mathbf{x})=\mathcal{V}_{2}(\mathbf{x})$; we ensure continuity along the boundary $\varphi \equiv 0$ by the additional SOS constraint

$$
\begin{aligned}
& -\mathcal{V}_{1}+\mathcal{V}_{2}+\varphi s_{\varphi, 1}-\varphi s_{-\varphi, 1} \in \Sigma[\mathbf{x}] \\
& -\mathcal{V}_{2}+\mathcal{V}_{1}+\varphi s_{\varphi, 2}-\varphi s_{-\varphi, 2} \in \Sigma[\mathbf{x}]
\end{aligned}
$$

where $s_{\varphi, i}, s_{-\varphi, i} \in \Sigma[\mathbf{x}]$ with $i \in\{1,2\}$ enforce that both $\mathcal{V}_{1}(\mathbf{x}) \leq \mathcal{V}_{2}(\mathbf{x})$ and $\mathcal{V}_{2}(\mathbf{x}) \leq \mathcal{V}_{1}(\mathbf{x})$ if $\varphi(\mathbf{x})=0$.

\section{Invariant sets under control}

The definition (7) and subsequent constraints $(9)-(11)$ apply equally to closed-loop controlled systems $\mathbf{f}_{\mathbf{K}}$ where $\mathbf{K}(\cdot)$ is linear or polynomial in $\mathbf{x}$. This observation alone, however, is insufficient to guarantee invariance under control as we have defined it above. We might now assume $\mathcal{U}$ to be defined as conjunction $\mathcal{U}=\left\{\mathbf{u} \mid p_{1}(\mathbf{u}) \leq 0, \ldots, p_{m}(\mathbf{u}) \leq 0\right\}$ with $\left(p_{l}\right)_{1 \leq l \leq m} \subset \mathbb{R}[\mathbf{u}] ;$ if, furthermore $\mathbf{K} \in \mathbb{R}[\mathbf{x}]^{p}$, we can state a necessary 
SOS constraint for invariance of $\Omega_{\lambda}$ under control $\mathbf{K}$ as

$$
-\left(p_{l} \circ \mathbf{K}\right)+(\mathcal{V}-\lambda) s_{l, \lambda^{\prime}} \in \Sigma[\mathbf{x}]
$$

where $\left(p_{l} \circ \mathbf{K}\right)(\mathbf{x})=p_{l}(\mathbf{K}(\mathbf{x}))$, with $s_{l, \lambda^{\prime}} \in \Sigma[\mathbf{x}]$ for all $1 \leq l \leq m$. The constraints (9)-(11) and (14), in fact, hold independently of each other and can be evaluated separately. As the subset-relation $\subset$ on the level sets $\left(\Omega_{\lambda}\right)_{\lambda>0}$ constitutes a total order, $\Omega_{\lambda_{\mathbf{K}}}$ with $\lambda_{\mathbf{K}}=\min \left\{\lambda, \lambda^{\prime}\right\}$ is an invariant subset of the region of attraction of $\mathbf{f}_{\mathbf{K}}(\cdot)$ under control $\mathbf{K}$.

3. $\mathcal{V}$-s-iteration

The ellipsoid $\mathcal{E}_{\rho}$ can be fitted inside the invariant set $\Omega_{\lambda}$ if

$$
-\left(\mathcal{V}_{i}-\lambda\right)+(P-\rho) s_{i, P} \in \Sigma[\mathbf{x}]
$$

with $s_{i, P} \in \Sigma[\mathbf{x}]$ for $i \in\{1,2\}$.

Note that we require $\mathcal{V}_{1,2}$ to be of some fixed degree. However, some constraints involve bilinear terms of the form $\mathcal{V}_{i} s_{(\cdot)}$ once $\mathcal{V}_{1,2}$ become decision variables. The $\mathcal{V}$-s-iteration uses a bisection approach of iteratively-alternating steps; a detailed discussion of the basic $\mathcal{V}$-s-iteration has been given by Chakraborty et al. [17. We extend the approach in order to incorporate control input constraints for a $\mathbf{K}(\cdot)$ given a priori and, later on, synthesize an optimal control feedback:

1a) Find $\lambda^{\circ}$ maximal such that 10 -11 hold for $\mathcal{V}_{1,2}$ fixed;

1b) Find $\lambda^{*}$ maximal such that (14) holds for $\mathcal{V}_{1,2}$ fixed;

2) Find $\rho^{\diamond}$ maximal such that 15 holds for $\mathcal{V}_{1,2}$ and $\lambda^{\dagger}=\min \left\{\lambda^{\diamond}, \lambda^{*}\right\}$ fixed;

3) Find $\mathcal{V}_{1}, \mathcal{V}_{2} \in \mathbb{R}[\mathbf{x}]$ of fixed degree such that $\left.(9)-15\right)$ hold for $\rho^{\diamond}$ and $\lambda^{\dagger}$ fixed.

The purpose of $\mathcal{E}_{\rho}$ here is twofold: first, to quantify the size of the provable invariant subset $\Omega_{\lambda}$ for each iteration; and second, to prevent the last step from yielding a smaller estimate than hitherto achieved.

\section{Control synthesis}

Until now, we have considered the control law to be given and fixed. Yet, we can further adapt our approach to find a suitable $\mathbf{K}$ in the attempt to enlarge the (estimated) region of attraction within the bounds imposed by the control input constrains. As the SOS constraints must be linear in the prospective control function, we require the control system to be in the companion form affine in $\mathbf{u}$, viz. $\dot{\mathbf{x}}=\mathbf{f}_{\mathbf{x}}(\mathbf{x})+\mathbf{f}_{\mathbf{u}}(\mathbf{x}) \mathbf{u}$ with $\mathbf{f}_{\mathbf{x}} \in \mathbb{R}[\mathbf{x}]^{n}, \mathbf{f}_{\mathbf{u}} \in \mathbb{R}[\mathbf{x}]^{n \times p}$, such that $\mathbf{f}_{\mathbf{K}}(\mathbf{x})=\mathbf{f}_{\mathbf{x}}(\mathbf{x})+\mathbf{f}_{\mathbf{u}}(\mathbf{x}) \mathbf{K}(\mathbf{x})$. The input constraints $\left(p_{l}\right)_{1 \leq l \leq m}$, too, must be 
linear in $\mathbf{u}$, that is, $p_{l}(\mathbf{u})=\mathbf{p}_{l}^{T} \mathbf{u}$ with $\mathbf{p}_{l} \in \mathbb{R}^{p}$, and $\mathbf{K}$ guarantees control-invariance of $\Omega_{\lambda^{\prime}}$ if

$$
-\mathbf{p}_{l}^{T} \mathbf{K}+\left(\mathcal{V}-\lambda^{\prime}\right) s_{l, \lambda^{\prime}} \in \Sigma[\mathbf{x}]
$$

with $s_{l, \lambda^{\prime}} \in \Sigma[\mathbf{x}]$ for all $1 \leq l \leq m$.

Remark The formulation of (16) as sum-of-squares constraints allows us to synthesize feedback laws of polynomial nature instead of feedback matrices, since both $\nabla \mathbf{f}_{\mathbf{K}}$ and $\mathbf{p}^{T} \mathbf{K}$ are linear in the coefficients of $\mathbf{K}$ regardless of the polynomial order.

In order to circumvent bilinearities, we execute again steps 1) and 2) for $\mathbf{K}$ fixed and incorporate a supplementary second-to-last step:

3a) Find $\mathbf{K} \in \mathbb{R}[\mathbf{x}]$ of fixed degree such that 100 -11] and (16) hold for $\mathcal{V}_{1,2}$ as well as $\rho^{\diamond}$ and $\lambda^{\dagger}$ fixed.

The old and new last step is once more computed for $\mathbf{K}$ fixed. The thus augmented iteration is performed by Algorithm 1 .

Remark Algorithm 1 could be modified by removing lines 11,14 and subsequently replacing the constraint (15) in line 4 by

$$
-\left(\mathcal{V}_{N, i}-\lambda^{\dagger}\right)+\left(\mathcal{V}_{N-1, i}-\lambda^{\dagger}\right) s_{i, \mathcal{V}} \in \Sigma[\mathbf{x}]
$$

with $s_{i, \mathcal{V}} \in \Sigma[\mathbf{x}]$ for all $i \in[1,2]$. The thus modified algorithm, to which we will refer as Algorithm $1 \mathrm{~b}$, does not need an ellipsoidal shape as input. Similar approaches have recently been employed for analysis of reachable sets of polynomial systems [26] and the region of attraction of hybrid systems [27. While Algorithm 10 allows the region-of-attraction estimate to grow in all directions rather than the one given by $\mathcal{E}$, we will see later that is unsuitable for the purpose of deep-stall recovery. Algorithm 1 and 13 are implemented in [28]. 


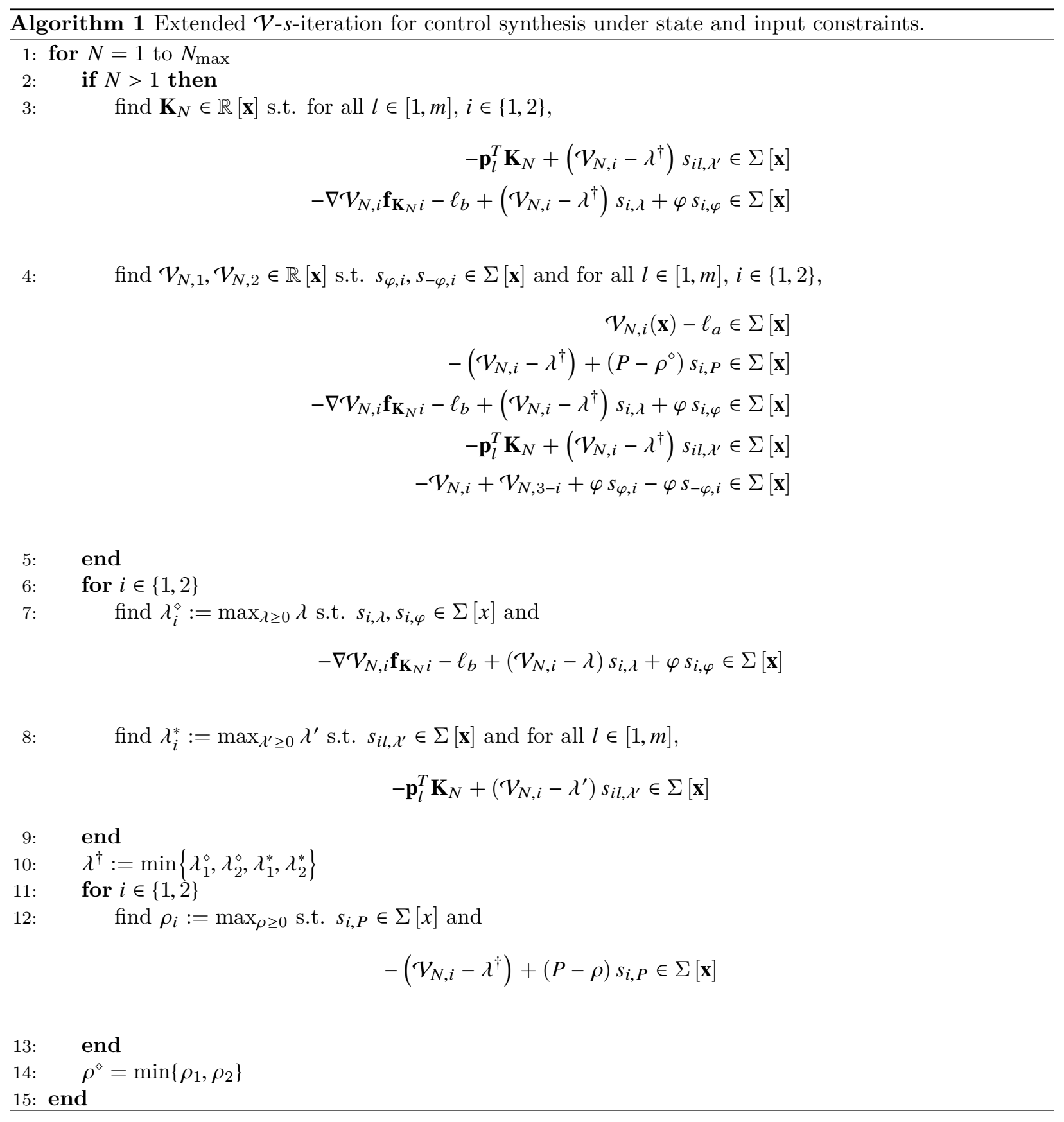




\section{Preliminary stability analysis}

In [21, we applied bifurcation theory as well as optimization techniques to derive the linear pitch-damping model in Eq. (3). We identified an optimal coefficient $C_{\mathrm{m} q} \approx 1.96$. In consequence, Fig. 3 shows the location and stability of longitudinal trim conditions (note that the choice of $C_{\mathrm{m} q}$ does not affect the location of stationary solutions as $q=0$ is a necessary conditions for trim), parametrized by the elevator deflection $\eta$. The black dot in Fig. 3 indicates the largest deflection, $\eta=6.5^{\circ}$, for which the aircraft enters a steep, nose-down descent; the minimal elevator deflection is $-60^{\circ}$. Shortly after stall, the aircraft encounters an unstable regime of stationary solutions with a family of limit cycles (Hopf bifurcation).

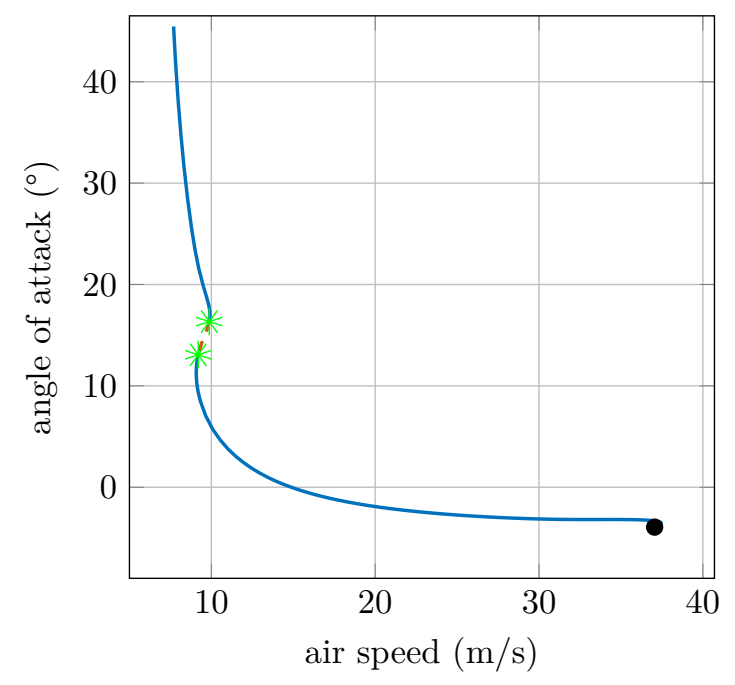

(a) Angle of attack $\alpha$ over air speed $V$.



(b) Pitch angle $\Theta$ over elevator deflection $\eta$.

Fig. 3 Trim conditions of longitudinal motion with unstable regimes (dashed) [21].

\section{Region of Attraction at Trim Point}

Where classical control synthesis relies upon linearized models, the region of attraction estimation provides knowledge about the limitations of the chosen control implementation. Unlike the safe set [see, e.g., 6, 7], which provides an exploratory study in order to estimate the abilities of the aircraft to be controlled, we study a region of attraction in the context of a given controller and the respective trim condition [20]. For the latter we choose a low-inclination gliding descent trim at $\eta_{\text {glide }}^{*}=-5^{\circ}$ (see Appendix A.

This section investigates the capability of various controllers to stably recover the aircraft from a deep-stall trim condition. We first consider a linear quadratic regulator, which could have been derived by classical control techniques. Later, we apply the SOS tools in order to derive a polynomial control law that improves stability and recovery of the vehicle. For both analysis and control synthesis, we scale the state vector $\mathbf{x}^{T}=\left[\begin{array}{llll}V_{\mathrm{A}} & \gamma_{\mathrm{A}} & q & \alpha\end{array}\right]$ by $\operatorname{diag}\left(10 \mathrm{~m} / \mathrm{s}, 45^{\circ}, 150^{\circ} / \mathrm{s}, 45^{\circ}\right)^{-1}$ and the input $\eta$ by $\left(80^{\circ}\right)^{-1}$ in order to normalize 
states and inputs. The scaled variables are henceforth denoted by $\tilde{\mathbf{x}}, \tilde{\eta}$, etc. The viable (unscaled) elevator inputs are given to $\mathcal{U}=\left[-60^{\circ}, 20^{\circ}\right]$ and represent the physical limits of the aircraft elevator. We further approximate the non-polynomial functions (sine, cosine, inverse) by Taylor series expansions and truncate high-order polynomial terms (Appendix A] in order to facilitate the resulting SOS problems [17].

Remark In [18, analysis of a six-state polynomial aircraft model has been considered, omitting inter alia the aircraft's speed from the state vector. Since deep-stall transition maneuvers span a large range of airspeeds [21], this approach would not be applicable here. Instead, we restrict this study to the four-state longitudinal motion (later, we will include the elevator deflection as a fifth state); however, as we consider piecewise-defined polynomial equations of motion of higher order, this model is in fact more complex than [18].

The aircraft longitudinal motion is commonly divided into short-period dynamics involving pitch rate and angle of attack as well as the long-period phugoid oscillation of airspeed and flight-path angle and often discussed separately, as if uncoupled. When discussing the region of attraction, we take into account the full, coupled 4-state model of Eqs. (1)-(4) but display the estimates as projections into either the phugoid $V_{\mathrm{A}}-\gamma_{\mathrm{A}}$ plane or short-period $\alpha-q$ plane. Details for all SOS computations are given in Appendix B

\section{A. Analysis of the Linear Quadratic Regulator}

A further but more advanced element of the classical linear toolbox is the renown linear quadratic regulator (LQR). Here, we minimize the quadratic cost function $\tilde{J}=\int_{0}^{\infty} \tilde{\mathbf{x}}(t)^{T} \tilde{\mathbf{Q}} \tilde{\mathbf{x}}(t)+\tilde{R} \tilde{\eta}(t)^{2} \mathrm{~d} t$ taking into account the linearized dynamics $\tilde{\mathbf{f}}\left(\tilde{\mathbf{x}}^{*}+\delta \tilde{\mathbf{x}}, \tilde{\eta}^{*}+\delta \tilde{\eta}\right) \approx \tilde{\mathbf{A}} \delta \tilde{\mathbf{x}}+\tilde{\mathbf{b}} \delta \tilde{\eta}$ in order to find a linear feedback. We obtain the optimal cost-to-go for an initial condition $\tilde{\mathbf{x}}_{0}=\tilde{\mathbf{x}}(0)$ as $\tilde{J}_{\text {opt }}=\tilde{\mathbf{x}}_{0}^{T} \tilde{\mathbf{S}} \tilde{\mathbf{x}}_{0}$, where $\tilde{\mathbf{S}}$ denotes the solution to the general Riccati equation with $(\tilde{\mathbf{A}}, \tilde{\mathbf{b}}, \tilde{\mathbf{Q}}, \tilde{R})$ and the LQR feedback is given as $\tilde{\eta}=\tilde{\mathbf{K}}_{\mathrm{LQR}}=-\left[\tilde{R}^{-1} \tilde{\mathbf{b}}^{T} \tilde{\mathbf{S}}\right] \tilde{\mathbf{x}}$. For weights $\tilde{\mathbf{Q}}=\mathbb{I}_{4 \times 4}, \tilde{R}=10$, the LQR feedback is synthesized to

$$
\tilde{\mathbf{K}}_{\mathrm{LQR}}=-0.1163 \tilde{V}_{\mathrm{A}}+0.3881 \tilde{\gamma}_{\mathrm{A}}+0.2412 \tilde{q}+0.0007 \tilde{\alpha}
$$

The control-invariant estimate of the region of attraction for $\tilde{\mathbf{K}}_{\mathrm{LQR}}$ is presented in Fig. 4 with the ellipsoidal shape $\mathcal{E}$ governed by $\tilde{P}=4 \tilde{V}_{\mathrm{A}}^{2}+4 \tilde{\gamma}_{\mathrm{A}}^{2}+\tilde{q}^{2}+\tilde{\alpha}^{2}$. Both $\mathcal{E}$ and the control inputs returned by the LQR feedback are illustrated in Fig. 4 as well.

Even after descaling, the LQR gain on the angle of attack is significantly smaller, diminishing its contribution to the overall control feedback. With the additional gains on airspeed and path inclination, the estimated region of attraction contains the aircraft's high-angle of attack conditions in the lower-left corner 


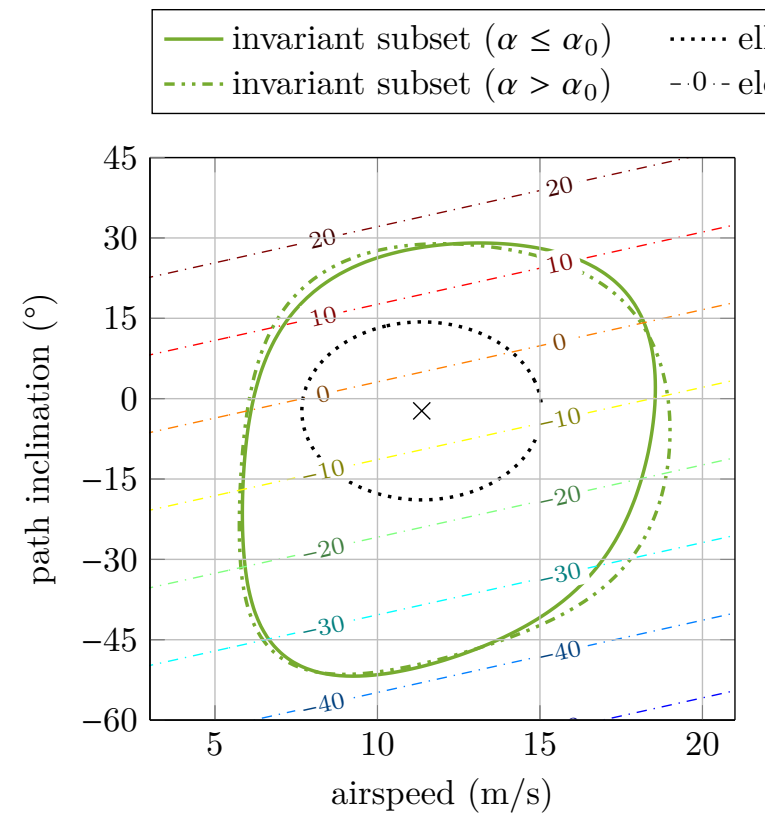

(a) Projection into phugoid plane. $\times$ trim condition $\left({ }^{\circ}\right)$

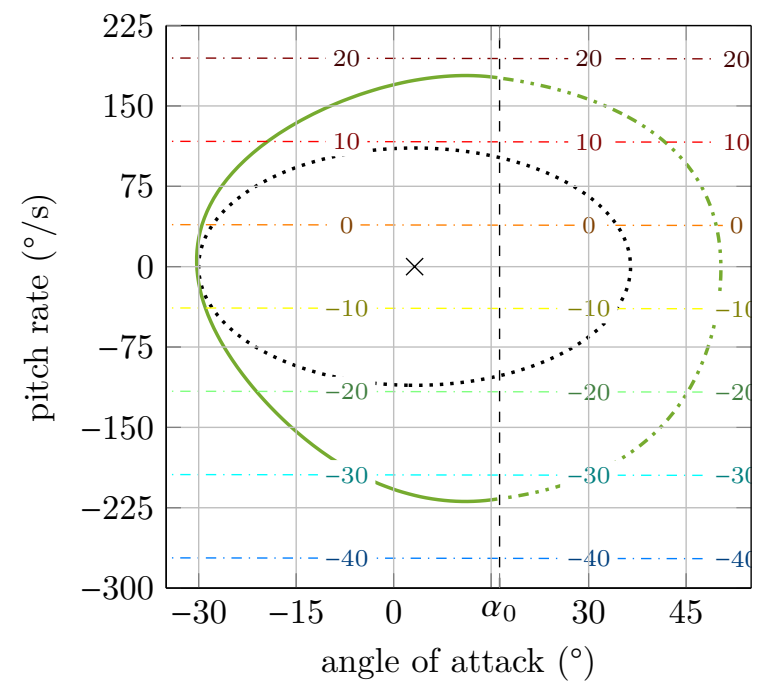

(b) Projection into short-period plane.

Fig. 4 Estimated control-invariant region of attraction of the linear quadratic regulator.

of the phugoid plane. Consequently, the depicted LQR feedback is able to stably recover from deep-stall.

In Fig. 5 we further compare this result with the estimated region of attraction obtained by Algorithm 1p, that is, without a predefined ellipsoidal shape. Black squares designate deep-stall trim conditions that are contained within the estimated invariant set of Algorithm 1 but not in the estimate of Algorithm $1 \mathrm{p}$, corresponding to elevator deflections from $-44^{\circ}$ to $-50^{\circ}$.

\section{B. Synthesis of polynomial control laws}

Until now, we have considered the control input to be determined by an a priori obtained state feedback law, which might have been designed by any means of control engineering. Synthesis of such a control law is subject to various objectives including desired closed-loop dynamics, disturbance rejection, and optimal reference tracking. From here on, we treat the feedback law as decision variables of sum-of-squares analysis rather than as part of the initial problem formulation. Thus, the feedback control is synthesized with the aim of enlarging the region of attraction, again quantified by the size of the ellipsoidal shape $\mathcal{E}$, towards deep-stall flight conditions. In the following, we subsequently derive a linear feedback, a polynomial feedback, and piecewise feedback comparable to gain-scheduling control approaches. Further details can be found in Appendix B

In order to reformulate the dynamics of Section II into the companion form, we take the elevator deflection 


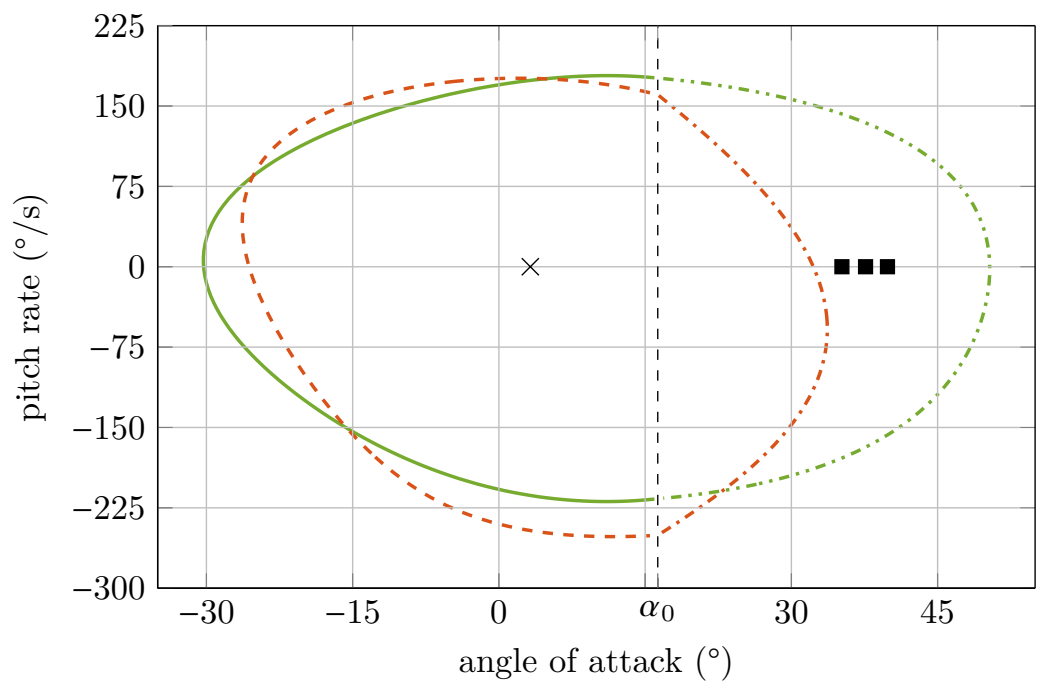

Fig. 5 Comparison with the estimate by Alg. $1 \mathrm{~b}$ (dashed) for the linear quadratic regulator.

$\eta$ to be a state and introduce the rate of the actuator, $d \eta$, as new input:

$$
\left[\begin{array}{c}
\dot{\mathbf{x}} \\
\dot{\eta}
\end{array}\right]=\left[\begin{array}{c}
\mathbf{f}_{\mathrm{EOM}}(\mathbf{x}, \eta) \\
0
\end{array}\right]+\left[\begin{array}{l}
0 \\
1
\end{array}\right] d \eta,
$$

where $\mathbf{f}_{\mathrm{EOM}}$ are the system dynamics of the previous sections. The thus extended state vector is denoted by $\mathbf{x}_{\eta}$ and scaled by $\operatorname{diag}\left(10 \mathrm{~m} / \mathrm{s}, 45^{\circ}, 150^{\circ} / \mathrm{s}, 45^{\circ}, 20^{\circ}\right)^{-1}$; the new input $d \eta$ is scaled by $\left(100^{\circ} / \mathrm{s}\right)^{-1}$. The viable (unscaled) actuator rate inputs are constrained to $|d \eta| \leq 200 \% \mathrm{~s}$ in order to ensure realistic actuator dynamics.

The role of the ellipsoid $\mathcal{E}$ and, in particular, the polynomial $P$ governing its shape for control synthesis deserves a further discussion. In the last step of the extended $\mathcal{V}$-s-iteration that is employed for both analysis and control synthesis, the ellipsoid $\left\{\mathbf{x} \mid P(\mathbf{x}) \leq \rho^{\diamond}\right\}$ serves as lower bound for the region of attraction estimate, both in size and shape. Recall further that each estimate $\left\{\mathbf{x} \mid \mathcal{V}(\mathbf{x}) \leq \lambda^{\circ}\right\}$ is itself invariant; if the feedback law is chosen prior to analysis, as in the preceding section, all invariant sets of the aircraft are predetermined by the closed-loop system dynamics. That is, we find a certain invariant set by guessing a Lyapunov function and computing its largest stable level set. With the feedback law being a decision variable of the control synthesis now, the selection of a control feedback actively shapes the resulting invariant set. Consequently, we expect the estimated region of attraction to follow the chosen ellipsoidal shape more closely and, therefore, we must carefully select its shape.

We will initially choose a polynomial $P$ of second order that results in an ellipsoid that is rotated with respect to the normal vector of the phugoid plane, in order to enhance recovery from deep-stall trim conditions, where air speed is exceptionally low and the path inclination is oriented steeply downwards. As the elevator 
deflection constitutes a state of Eq. (18), the constraints $\eta \in\left[-60^{\circ} ; 20^{\circ}\right]$ form asymmetric boundaries. Hence, to ensure recovery from deep-stall trim of conditions with large negative elevator deflections is challenging. We will therefore employ an asymmetrically defined quasi-ellipsoidal shape governed by a fourth-order polynomial.

\section{Linear feedback control}

We start with synthesizing a linear feedback law $d \eta=\mathbf{K}_{\text {lin }}=\mathbf{G} \mathbf{x}$, where $\mathbf{G} \in \mathbb{R}^{5 \times 5}$ is a decision variable of the sum-of-squares program. Fig. 6 shows the estimated region of attraction for the synthesized linear control feedback. The ellipsoidal shape $\mathcal{E}$ is governed by

$$
\tilde{P}=220 \tilde{V}_{\mathrm{A}}^{2}-360 \tilde{V}_{\mathrm{A}} \tilde{\gamma}_{\mathrm{A}}+100 \tilde{\alpha}^{2}+25 \tilde{\eta}^{2}+220 \tilde{\gamma}_{\mathrm{A}}^{2}+100 \tilde{q}^{2}
$$

and rotated with respect to the phugoid plane. The synthesized linear feedback law is illustrated in Fig. 6 as contour plots with respect to states and elevator deflection.



(a) Phugoid plane.

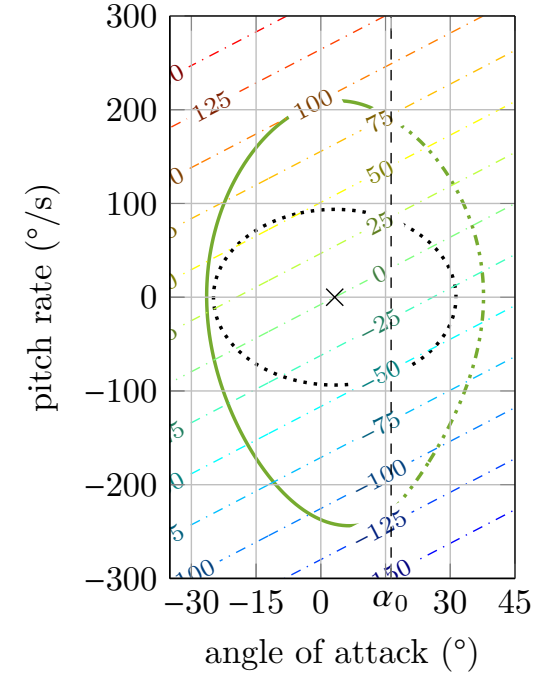

(b) Short-period plane. $\times$ trim condition

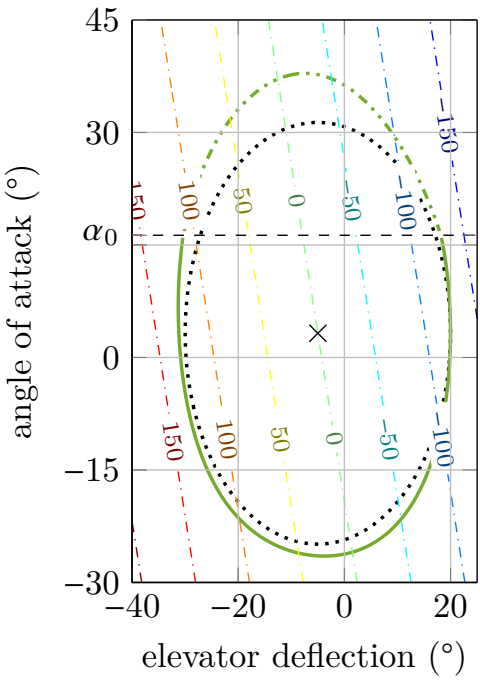

(c) Elevator-input plane.

Fig. 6 Region of attraction of the synthesized linear control feedback matrix.

The synthesized linear feedback maintains a region of attraction that contains initial conditions of a wide range of airspeeds, path inclination, and angles of attack. In the phugoid and short-period plane, the invariant set only loosely follows the ellipsoidal shape, leading to an enlarged region of attraction. However, the ellipsoidal shape starkly affects the elevator deflection, as the dynamics of the actuator are decoupled, and the upper bound constrains both positive and negative deflections. Thus, negative elevator deflections 


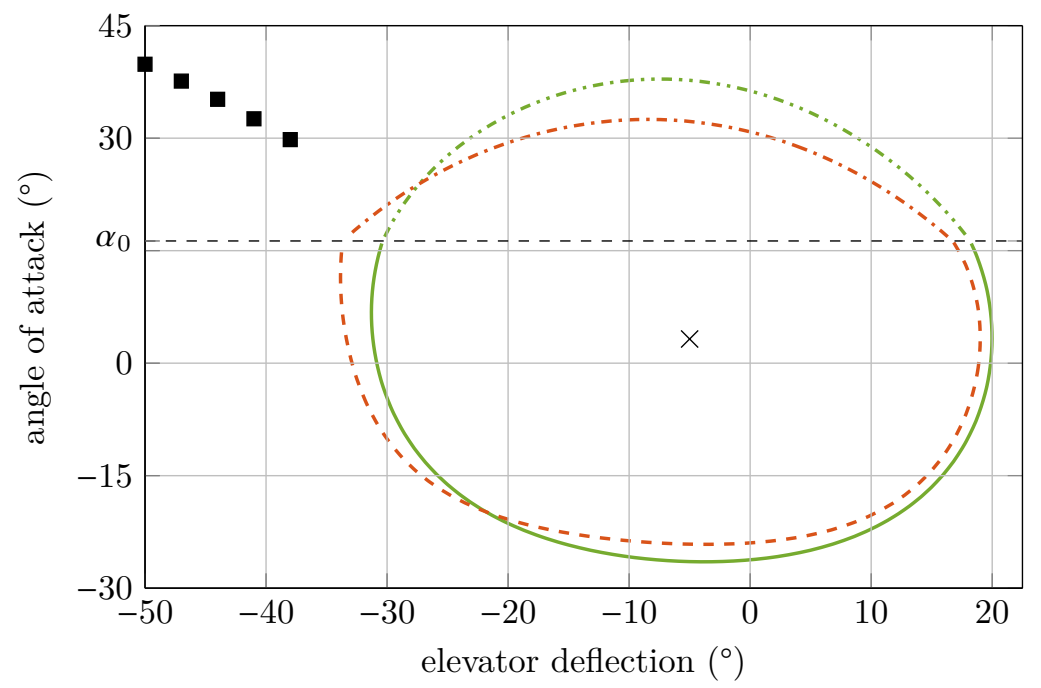

Fig. 7 Comparison with the result of Alg. 1 b (dashed) for linear control synthesis.

observed in deep-stall flight are not contained by the estimated region of attraction of this linear feedback controller. Nor are those flight conditions contained by the estimated region of attraction with a feedback synthesized by Algorithm 1 $\mathrm{b}$, as shown in Fig. 7 Here, black squares correspond to deep-stall trim conditions with elevator deflections ranging from $-38^{\circ}$ to $-50^{\circ}$. In the following, we will use quasi-ellipsoids of higher order in order to shape the synthesized controller and the estimated region of attraction towards deep-stall conditions. Given the asymmetric elevator constraints, we propose a new algorithm for deep-stall recovery synthesis.

\section{Polynomial feedback control}

Indeed, sum-of-squares control synthesis benefits from its ability to synthesize polynomial feedback laws that are not represented by linear matrices. Whereas the candidate Lyapunov function is conveniently represented by a polynomial without linear coefficients and of even degree to facilitate positivity of the Lyapunov function, it seems reasonable to have a polynomial feedback law without constant terms and of odd degree. Here, we choose a polynomial $d \eta=\mathbf{K}_{\text {poly }}$ with linear, quadratic, and cubic terms. Furthermore, to maintain a region of attraction including large negative elevator deflections, we select an asymmetric quasi-ellipsoidal shape $\mathcal{E}$ that is governed by $\tilde{P}_{\text {poly }}$ given in Appendix $\mathrm{C}$ The estimated region of attraction for the synthesized third-order control feedback is shown in Fig. 8 .

The differences between third-order (cubic) and first-order (linear) feedback laws can well be obtained from the isolines, that is, the contour lines of equal actuator rate inputs. While the input increases (or decreases) faster with increasing distance to the trim point, the course of the isolines also varies starkly within different sections of the state space. Moreover, the invariant set seems to be embedded into the contour lines 


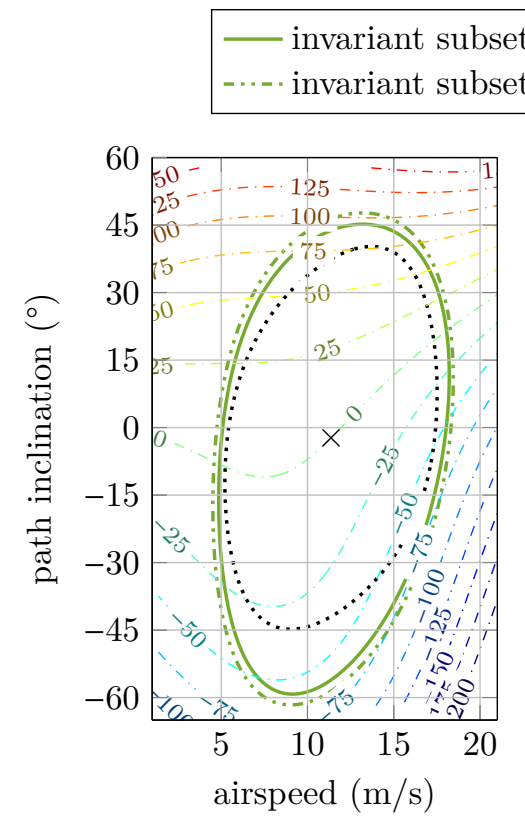

(a) Phugoid plane.

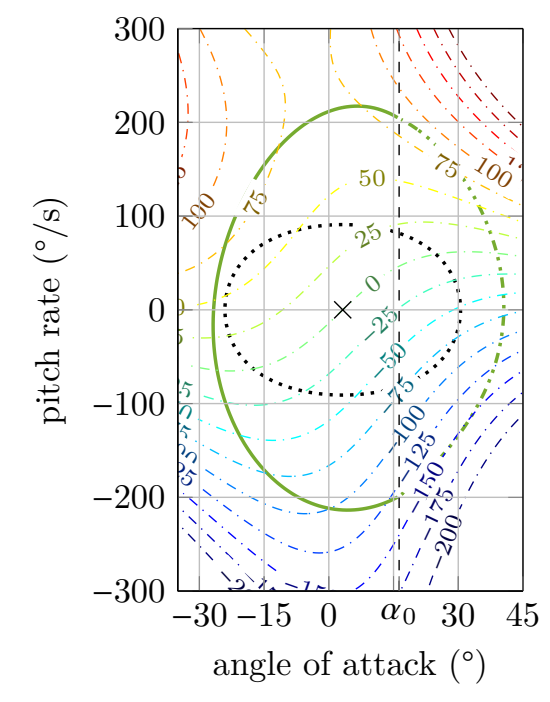

(b) Short-period plane.

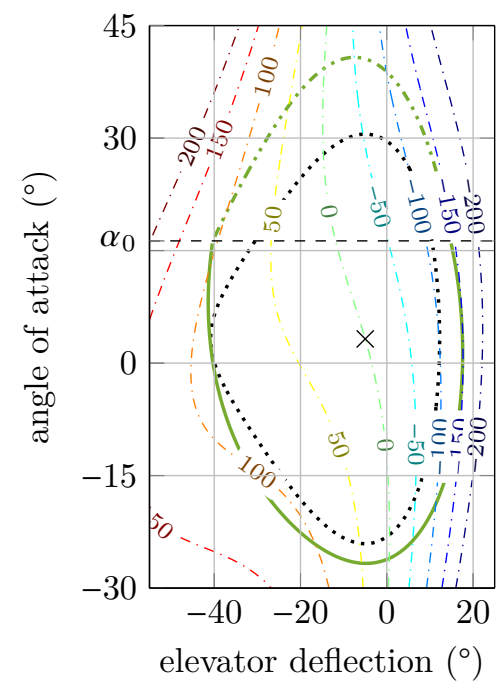

(c) Elevator-input plane.

Fig. 8 Region of attraction of the synthesized polynomial control feedback law.

of equal feedback.

The asymmetric quasi-ellipsoid pushes the boundary of the invariant set towards larger negative elevator deflections, without violating the upper constraint. However, deep-stall trim conditions with their angles of attack of $\geq 30^{\circ}$ are not contained by the estimated region of attraction.

\section{Piecewise feedback control}

It seems desirable to have alternative control laws for high and low angles of attack in order to adapt for changed dynamics beyond stall. With the aerodynamic model defined piecewise, it is convenient to synthesize a piecewise polynomial control law for the same regions, that is,

$$
d \eta=\mathbf{K}_{\mathrm{pw}}(\mathbf{x})=\left\{\begin{array}{cc}
\mathbf{K}^{\text {pre }} & \text { if } \alpha \leq \alpha_{0} \\
\mathbf{K}^{\text {post }} & \text { otherwise }
\end{array}\right.
$$

where $\mathbf{K}^{\text {pre }}, \mathbf{K}^{\text {post }}$ are third-order polynomials in $\mathbf{x}$. Note that we don't require a boundary condition, as we command a change of deflection, but could enforce equality of $\mathbf{K}^{\text {pre }}$ and $\mathbf{K}^{\text {post }}$ along $\alpha \equiv \alpha_{0}$ similar to 12. 13 . When synthesizing polynomial feedbacks for a control-invariant region of attraction spanning both low and high angles of attack, neither may violate state and input constraints within their respective domains. Fig. 9 shows the estimated region of attraction for the synthesized piecewise third-order control 
feedback. The ellipsoidal shape $\mathcal{E}$ is extended towards the section of high angles of attack and large negative elevator deflections and therefore governed by $\tilde{P}_{\mathrm{pw}}$ detailed in Appendix $\mathrm{C}$ For the sake of legibility of the phugoid-plane projection, we only show the contour lines of equal control feedback for the low angle of attack law.

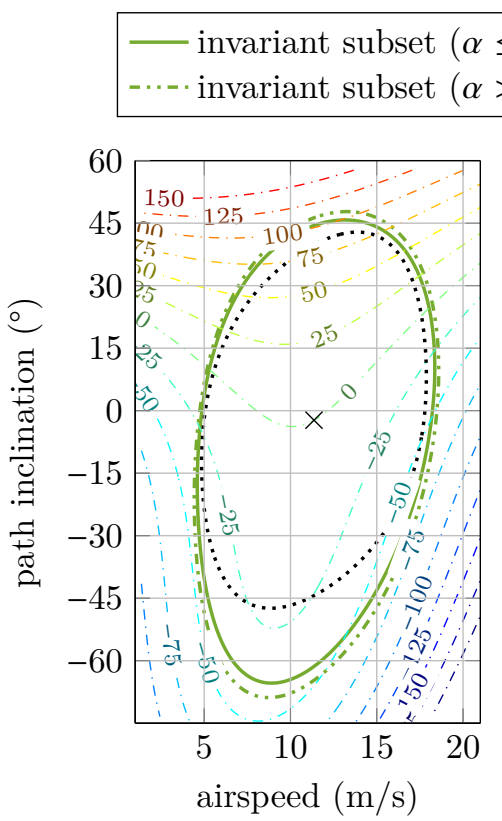

(a) Phugoid plane.

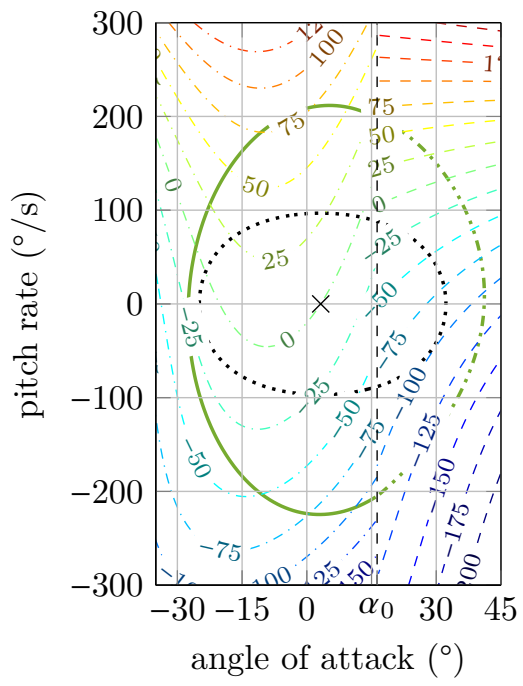

(b) Short-period plane.
- 0-- actuator rate $\left(\% / \mathrm{s}, \alpha \leq \alpha_{0}\right)$ - 0 - actuator rate $\left(\% / \mathrm{s}, \alpha>\alpha_{0}\right)$

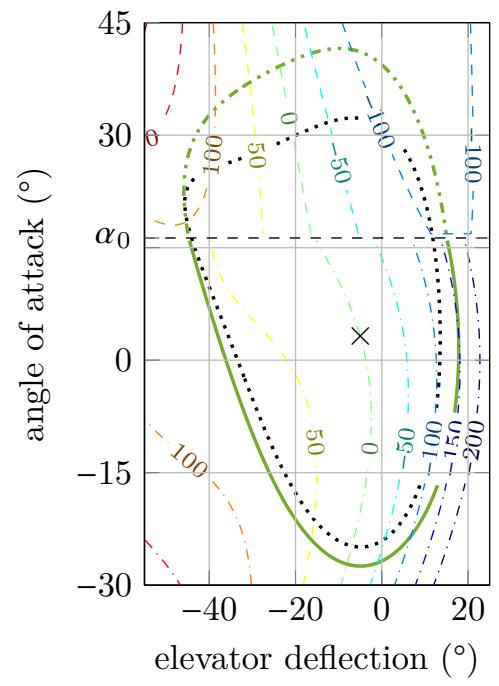

(c) Elevator-input plane.

Fig. 9 Region of attraction of the synthesized piecewise polynomial control feedback law.

\section{Discussion}

We employed the sum-of-squares framework in order to synthesize linear, polynomial, and piecewise polynomial control feedback laws that, by design, grant an enlarged region of attraction subject to constraints on the deflection and rate of change of the elevator. In Algorithm 1 as discussed in the beginning, the choice of the shape $P$ proves to be crucial for the form of the synthesized feedback law and thus for the shape of the resulting provable invariant set. Alternatively proposed iteration schemes, such as Algorithm 1 $\mathrm{p}$, aimed to increase the volume of the region-of-attraction estimate directly rather than enlarging an inscribing polynomial shape function [26, 27, 29, 30]. In particular, Algorithm 10 replaces the ellipsoidal shape by a constraint enforcing that each prior estimate is nested in the next estimate, thus removing the necessity of choosing a shape function. However, as demonstrated in Figs. 5 and 7 a control feedback that maximizes the region of attraction in a general sense is not necessarily able to recover from the particular deep-stall flight conditions. We want instead to specifically enlarge the region of attraction towards the desired states. Therefore, we need sufficient directional information to shape the region of attraction. 
Remark During the iteration the surface $\mathcal{E}_{\rho}$ and the input constraints form lower and upper bounds, respectively, for the computation of a new feedback law $\mathbf{K}$. As the deflection constraint has been particularly asymmetric $\left(-60^{\circ}\right.$ to $\left.20^{\circ}\right)$, we made use of increasingly complicated, asymmetrically shaped quasi-elliposidal surfaces.

We were able to synthesize feedback laws for recovery from flight conditions at increasingly steep descents and low airspeeds without violating the elevator deflection constraint. However, if the generated invariant sets do not contain the deep-stall flight conditions, which is the case, this is due to the choice of $P$ as a parameter of the $\mathcal{V}$-s-iteration rather than the form of the control feedback. Despite a large number of iterations, when these bounds finally converge and the iteration terminates (see Table 2 in Appendix B), we have not succeeded in reaching the deep-stall flight conditions with the resulting invariant set under the synthesized control. In the next section, we will therefore propose an alternative algorithm that directly formulates the SOS control synthesis as an infinite-horizon reachability problem.

Remark Sum-of-squares programming is in practice limited by the size of the resulting semidefinite problems, which in turn is a function of both the number of state variables and polynomial degree, and scales badly with increasing degree [31. A further partitioning of the state-space into piecewise defined polynomials could help reduce the polynomial degree necessary to accurately represent aircraft dynamics and thus the underlying matrix size. Analysis of such multi-variate spline models requires additional decision variables in Eqs. 10-11]. In [32, we proposed an efficient algorithm for multi-variate splines that detects the active boundaries in line 7 of Algorithm 1 and thus reduces the total number of decision variables. On the other hand, the number of constraints in line 4 of the same algorithm, that is, the number of conditions (12)- 13 , increases with the number of active boundaries, too. Hence, the partitioning into polynomial submodules is subject to this trade-off.

\section{Deep-stall Recovery}

Stabilization of deep-stall trim conditions via SOS control synthesis, as the previous section revealed, remains subject to careful selection of the ellipsoidal shape $\mathcal{E}$. Defining a polynomial surface, in particular in higher dimensions and larger-than-quadratic order, is a nontrivial task (see also Appendix C for a sumof-squares procedure for quasi-ellipsoids based on a selection of points). On the other hand, deep-stall recovery is often formulated as finite-horizon problem, namely, as part of a multi-mode paradigm where the flight controller switches to a local feedback after recovery. In this section, we modify the control synthesis into a simplified backwards reachability scheme, where the ellipsoidal shape is replaced by a target state whence recovery is to be ensured. An alternative approach for a finite-horizon backwards reachability analysis 
was presented in [26]. As Algorithm 10, this approach lacks any directional information for enlarging the closed-loop reachable set under the control law to be synthesized. We further provide a numerical comparison of deep-stall recovery by all presented feedback laws in the time domain.

\section{A. Infinite-horizon reachability control synthesis}

Denote the target state by $\mathbf{x}_{1}$ and the distance vector from the nominal trim by $\overline{\mathbf{x}}=\mathbf{x}_{1}-\mathbf{x}^{*}$. The target state is contained in the region of attraction of the nominal trim condition with control feedback $\mathbf{K}$ (i.e., $\mathbf{x}_{1}$ is backwards reachable from $\mathbf{x}^{*}$ in infinite time), if there is a function $\mathcal{V}(\cdot)$ and level set $\lambda$ such that $\Omega_{\lambda}$ is a control-invariant region of attraction estimate (cf. Eqs. $10-(14)$ ) and $\mathbf{x}_{1} \in \Omega_{\lambda}$, that is, $\mathcal{V}(\overline{\mathbf{x}}) \leq \lambda$. Obtaining such $\mathbf{K}, \mathcal{V}(\cdot)$, and $\lambda$ requires again bisection: Given $\mathcal{V}(\cdot)$ and $\lambda$ such that $10-14$ hold, we define the degree of reachability $\vartheta^{\diamond}$ as the maximal distance ratio $\vartheta$ such that

$$
\mathcal{V}(\vartheta \overline{\mathbf{x}}) \leq \lambda
$$

and observe that $\mathbf{x}_{1}$ can be recovered if $\vartheta^{\diamond} \geq 1$. For robustness, it might be desirable to have $\mathbf{x}_{1}$ well inside the interior of $\Omega_{\lambda}$, that is, the degree of reachability is strictly larger than one or even satisfies a chosen margin. The $\mathcal{V}$-s-iteration for backwards reachability is then formulated as follows:

1) Find $\lambda^{\circ}, \lambda^{*}$ maximal such that $10-11$ as well as 14 hold for $\mathcal{V}$ and $\mathbf{K}$ fixed;

2) Find $\vartheta^{\diamond}$ maximal such that 20$]$ holds for $\mathcal{V}$ and $\mathbf{K}$ as well as $\lambda^{\prime}=\min \left\{\lambda^{\diamond}, \lambda^{*}\right\}$ fixed;

3a) Find $\mathbf{K} \in \mathbb{R}[\mathbf{x}]$ of fixed degree such that 10 -11] and (16) hold for $\mathcal{V}$ as well as $\vartheta^{\diamond}$ and $\lambda^{\prime}$ fixed;

3b) Find $\mathcal{V} \in \mathbb{R}[\mathbf{x}]$ of fixed degree such that (9)-14) and (20) hold for $\mathbf{K}$ as well as $\vartheta^{\diamond}$ and $\lambda^{\prime}$ fixed.

Instead of $\mathcal{E}_{\rho}$ before, the degree of reachability $\vartheta^{\diamond}$ ensures that, in the last step, the region of attraction estimate grows towards the target state $\mathbf{x}_{1}$, that is, the degree of reachability increases. The thus modified $V$-s-iteration is performed by Algorithm 2. Note that, unlike finite-horizon reachability the obtained feedback law $\mathbf{K}$ stabilizes the target trim condition beyond recovery, too. Line 11 of Algorithm 2 cannot be solved as the sum-of-squares problem $(20)$ is not linear in $\vartheta$ for functions $\mathcal{V}(\cdot)$ of mixed polynomial degree, but is efficiently obtained using a nonlinear solver such as MATLAB's fmincon.

We choose now one of the stable deep-stall trim conditions as target state, namely, that at $\eta_{1}=-40^{\circ}$ (see Appendix A. Again, states and inputs are scaled to $\tilde{\mathbf{x}}, \tilde{d \eta}$, etc. and subject to state and input constraints. For the sake of a demonstration here, we choose a single function-candidate $\mathcal{V}(\cdot)$ as well as a linear control feedback. Fig. 10 illustrates the estimated region of attraction, a five-dimensional surface, as slices projected into the phugoid plane; for this purpose, we assign the free parameters as $\alpha=t \bar{\alpha}, q=t \bar{q}$, and $\eta=t \bar{\eta}$ and use the ratio $t$ for the out-of-plane drawing axis. 


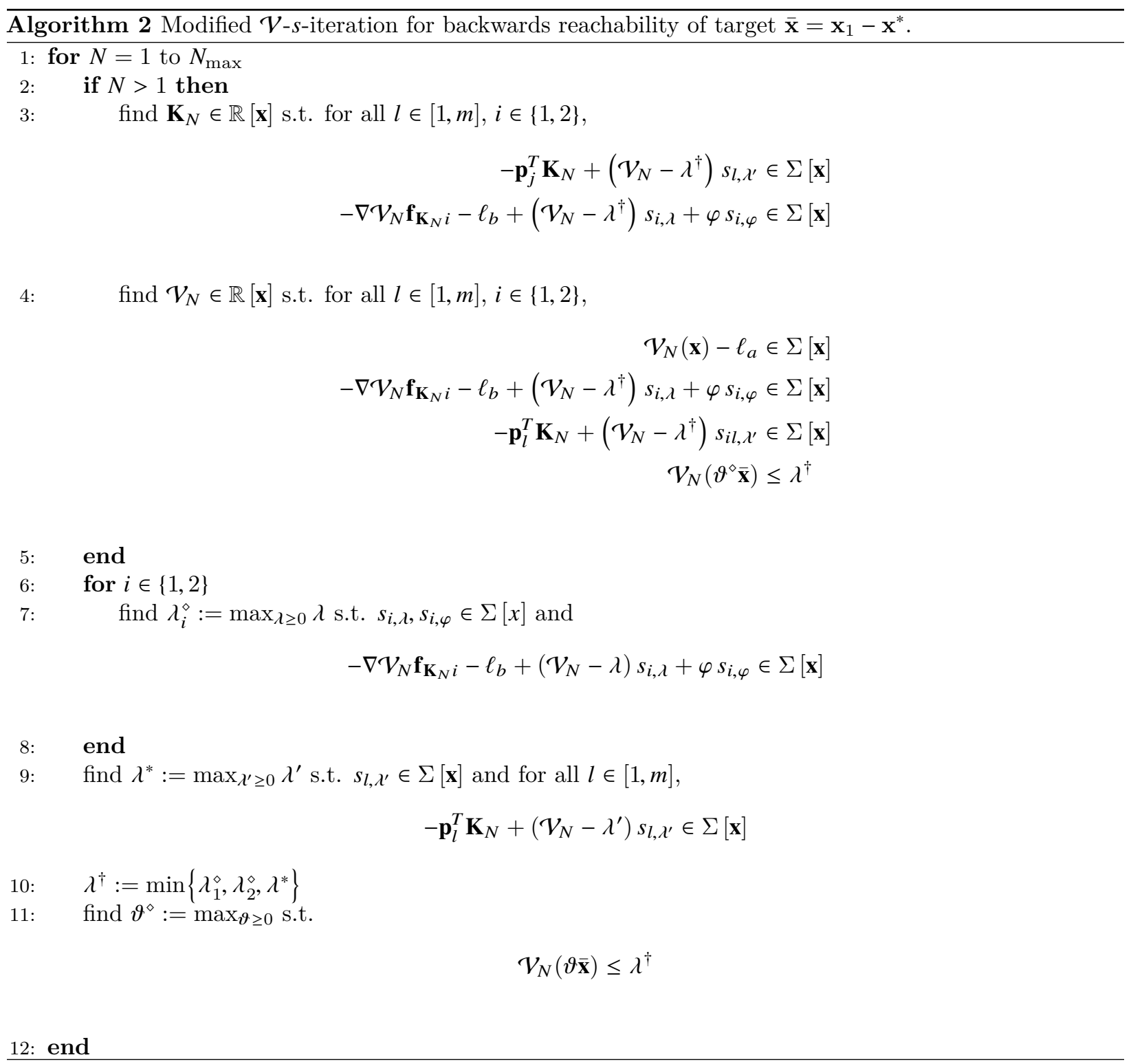




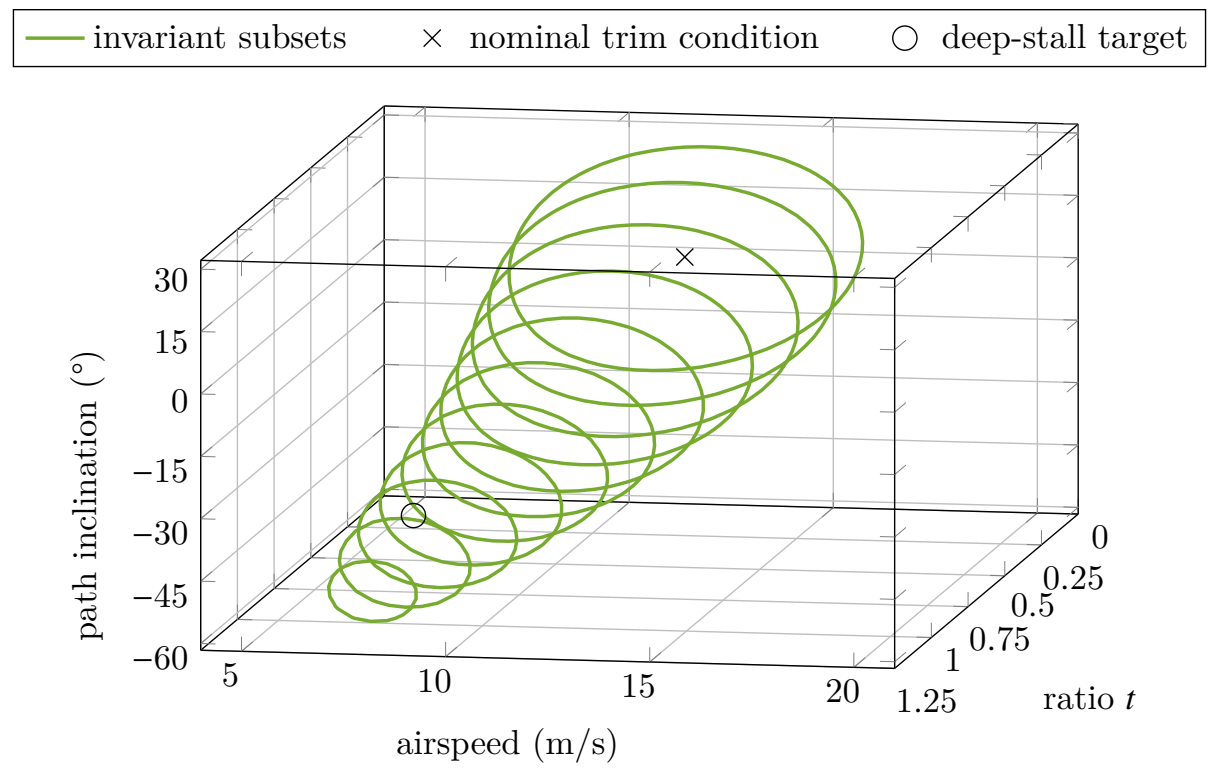

Fig. 10 Region of attraction of a linear control feedback law, synthesized by backwards reachability of the deep-stall target.

With a terminal degree of reachability of $\vartheta^{\diamond}=1.3027$, the synthesized feedback law robustly recovers the aircraft from the deep-stall target into nominal flight. Note that the invariant subset is shaped mainly around the trajectory from $\mathbf{x}_{1}$ to $\mathbf{x}^{*}$, according to the objective, rather than growing as large as possible. This outcome is further illustrated in Fig. 11, which depicts the estimated regions of attraction in the course of the 39 iterations. With increasing iterations, the region of attraction estimate stretches towards the target flight condition until it contains $\mathbf{x}_{1}$ (i.e., $\vartheta^{\diamond} \geq 1$ ).

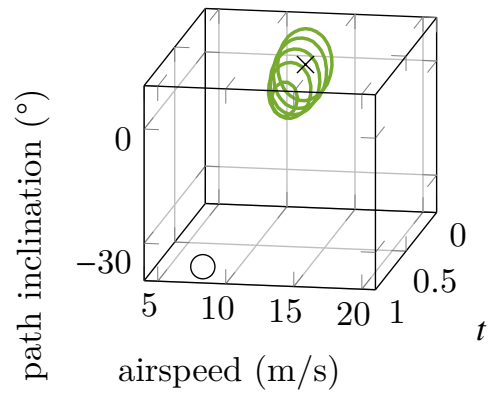

(a) 5 iterations $\left(\vartheta_{5}^{\diamond}=0.2179\right)$.

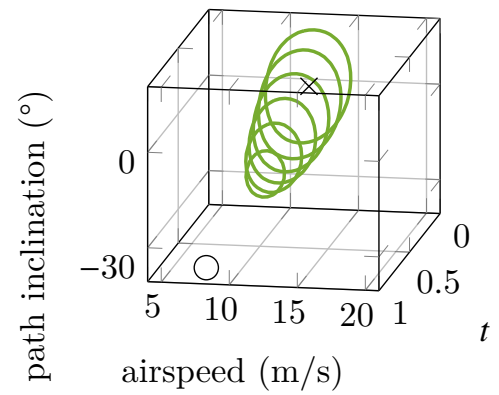

(b) 15 iterations $\left(\vartheta_{15}^{\diamond}=0.5486\right)$.

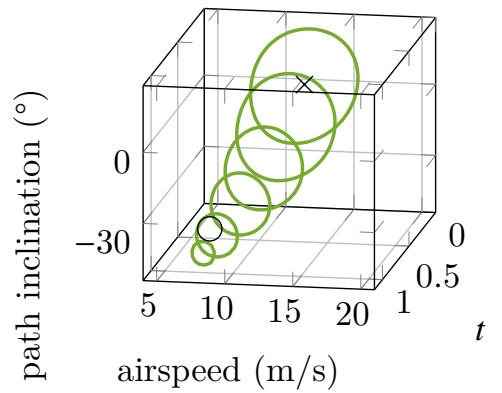

(c) 25 iterations $\left(\vartheta_{25}^{\diamond}=1.1841\right)$.

Fig. 11 Region of attraction estimates during iteration of the backwards-reachability control synthesis.

\section{B. Numerical comparison}

We consider recovery from the deep-stall trim condition $\mathbf{x}_{1}$, given in Appendix A, and simulate the closed-loop behaviour for the feedback laws discussed in this study. Fig. 12 compares recovery under the 
control synthesized for reachability to the previous synthesized linear, polynomial, and piecewise polynomial feedback laws, as well as to the LQR feedback. In addition, the closed-loop response is compared to a single-rate damping law, $\tilde{\eta}=\tilde{k}_{\eta q} \tilde{q}$, where $\tilde{k}_{\eta q}=1$ is a positive, proportional gain on the (scaled) pitch rate. All trajectories have been computed using the non-polynomial longitudinal equations of motion given in Eqs. (1)-(4) rather than the polynomial approximations employed for the SOS iterations.

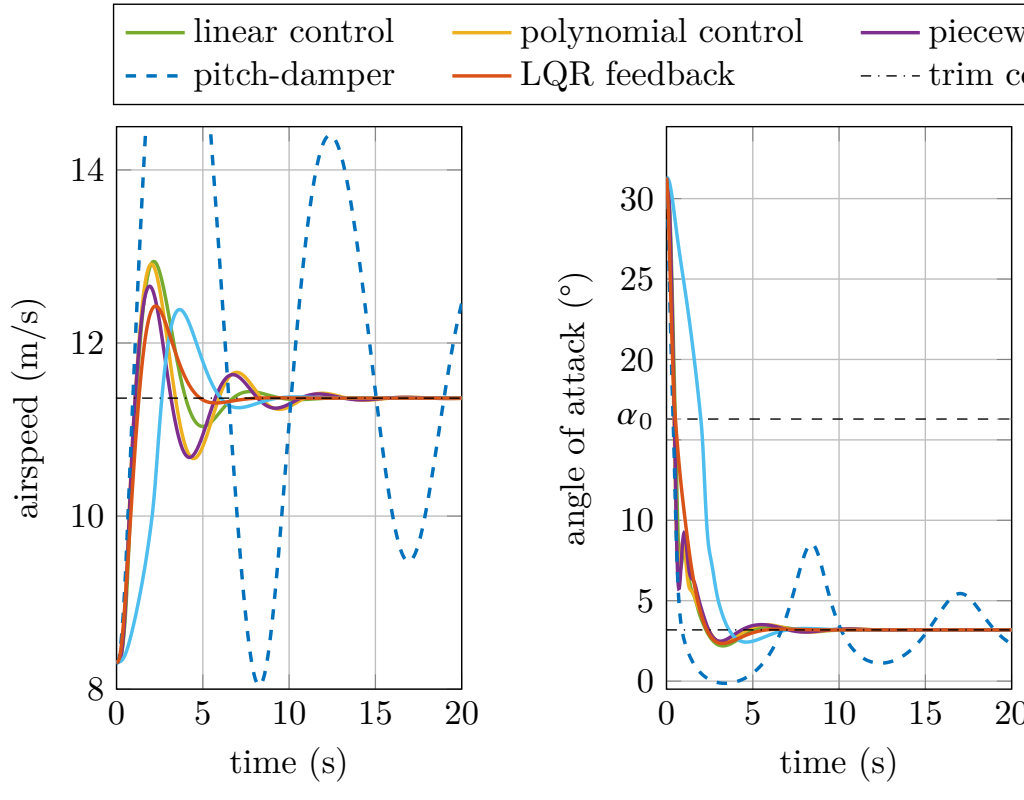

(a) Airspeed $V_{\mathrm{A}}$.

(b) Angle of attack $\alpha$.

Fig. 12 Comparison of time responses starting in $\mathrm{x}_{1}$.

The LQR controller, as described in Section III recovers stably and fast; the pitch-damper (dashed) eventually recovers though delayed and with large oscillations. As for the control laws of Section B. the objective of synthesis has been an enlarged region of attraction rather than closed-loop performance; consequently, the recovery is performed rather slowly and with considerable overshoot (in particular for the polynomial and piecewise polynomial feedback laws). The control law synthesized for reachability of the deep-stall condition also leads to a slow but straight recovery.

Remark An extended SOS control synthesis improving performance measures in addition to enlarging the region of attraction and/or ensuring backwards reachability could be achieved by further maximizing an exponential stability gain in Eqs. 10 and (11), viz.

$$
-\nabla \mathcal{V} \mathbf{f}_{\mathbf{K}}-\zeta \mathcal{V} \in \Sigma[\mathbf{x}]
$$

where $\zeta>0$ is subject to maximization once the region of attraction is sufficiently enlarged. 


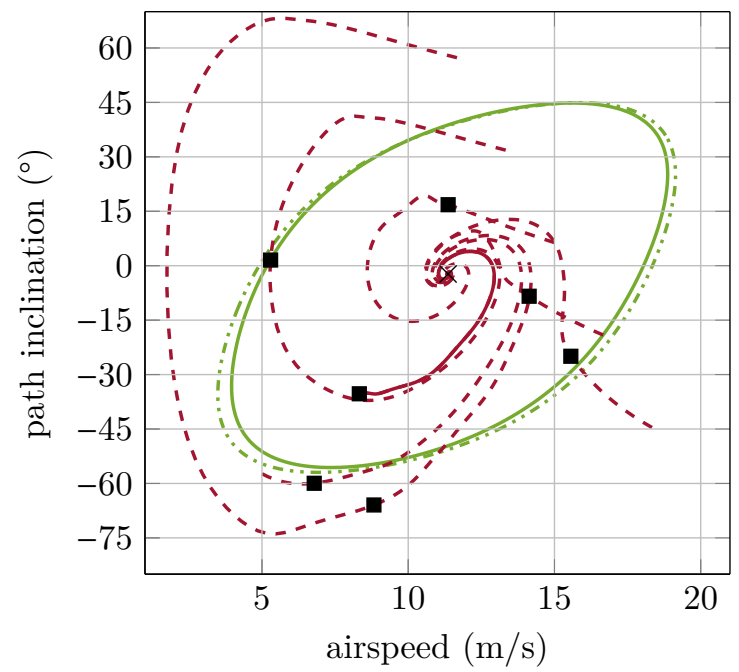

(a) Linear control law.

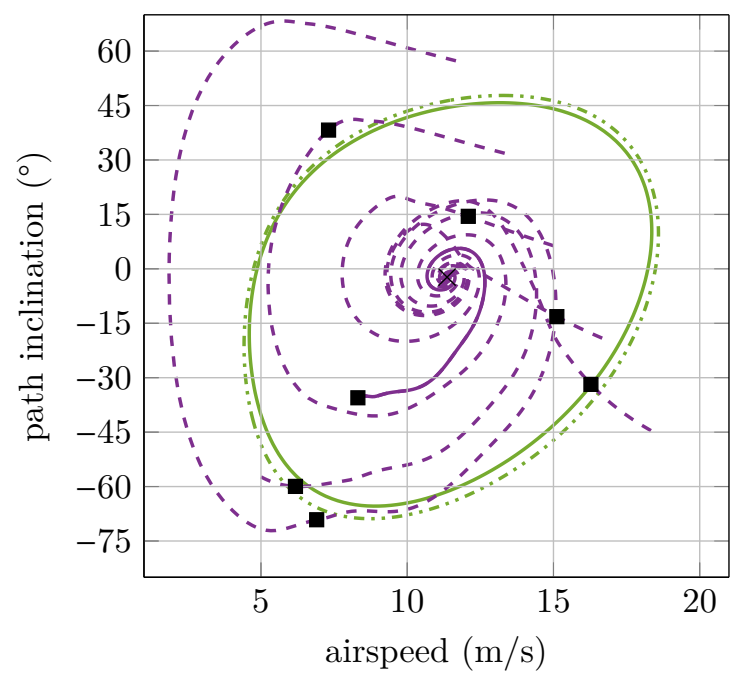

(c) Piecewise control law.

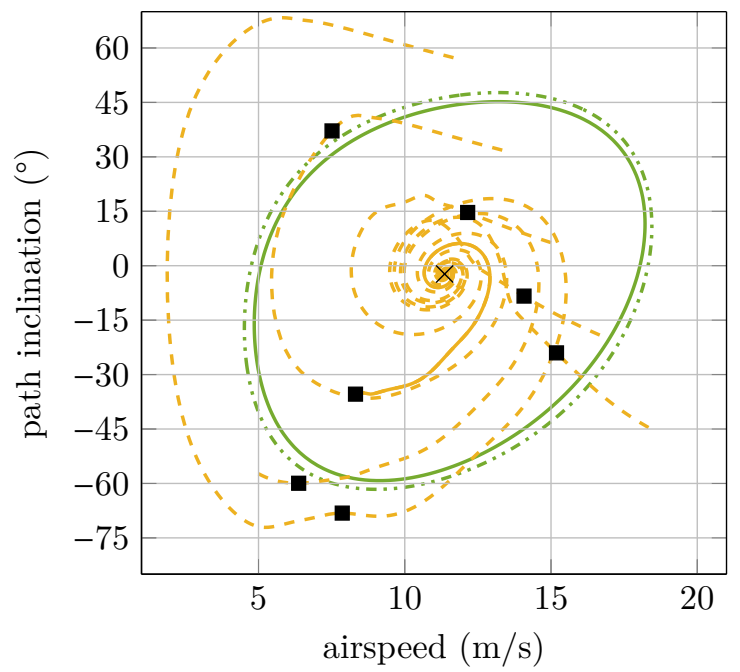

(b) Polynomial control law.

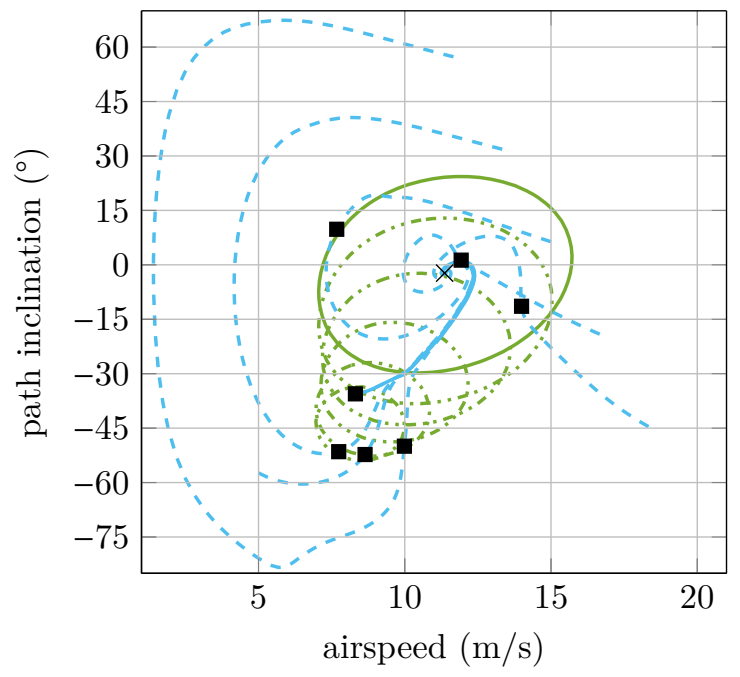

(d) Reachability-synthesis law.

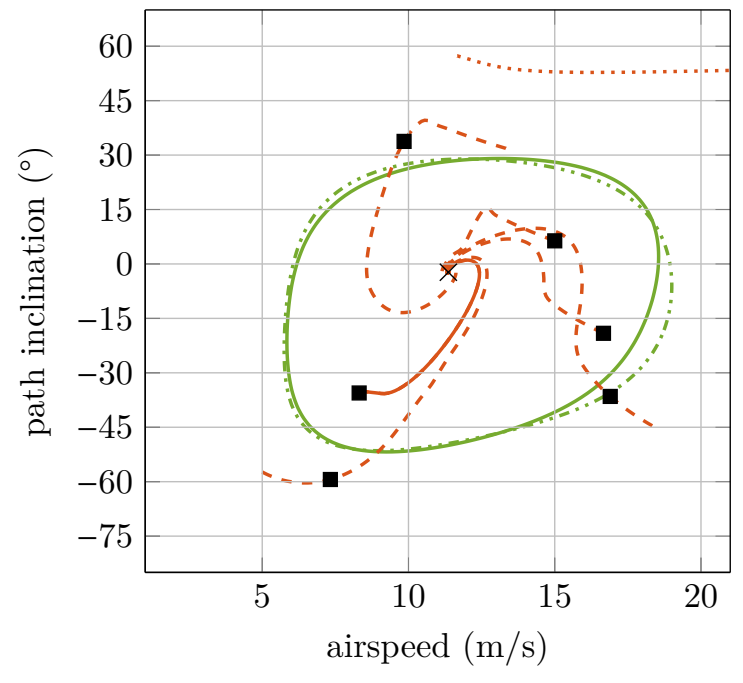

(e) LQR feedback law.

Fig. 13 Time responses and region of attraction estimates in the phugoid plane. 
In Fig. 13, we detail trajectories starting in various deep-stall flight conditions in the phugoid plane and additionally show projections of the respective region-of-attraction estimates. The response for the trim condition $\mathbf{x}_{1}$ is drawn in solid; the other responses (dashed) have been computed for non-trim conditions given in Appendix A. In addition, for each trajectory, the point it enters the region of attraction estimate is marked by a black square (due to the projection into the phugoid plane, these points might not necessarily appear inside the ellipsoid). For the feedback law synthesized by backwards reachability, further out-of-plane slices of the estimated region of attraction are projected into the phugoid plane as well. Except for one trajectory (dotted) of the LQR feedback, all controllers are able to recover from each deep-stall condition into the gliding trim condition. However, for the high-inclination conditions $\mathbf{x}_{6}$ and $\mathbf{x}_{7}$ this has partially led to saturations of the elevator deflection and its rate of change. Therefore, even though the saturation has not prevented recovery, these conditions could not have been included in any region of attraction estimate subject to the imposed constraints in the methodology presented in this paper. In the other cases, the fact that stable recovery trajectories lie outside the estimates highlights the inevitable conservatism of any sum-of-squares analysis approach.

\section{Conclusion}

Sum-of-squares techniques provide exact certificates for stability and the region of attraction, but the application to accurate, full-envelope aircraft models such as multivariate splines is computationally demanding. Simple piecewise polynomial models, on the other hand, accommodate the aerodynamic coefficients well in both domains of low and high angles of attack, while only moderately increasing the costs of sum-of-squares analysis. In this paper, we have applied sum-of-squares techniques to a fixed-wing aircraft model in order to verify stable recovery from deep-stall. As an intermediate result, we verified that classical LQR feedback control of the elevator deflection is sufficient for recovery without violation of input constraints. We then further extended the sum-of-squares analysis for control synthesis, using the actuator rate instead of deflection. Here, the choice of an ellipsoidal shape function for the sum-of-squares program turned out to be crucial, yet the selection was challenged by asymmetric constraints on the elevator deflection. Although none of the synthesized linear, polynomial, and piecewise polynomial feedback laws could be verified to recover the aircraft from its deep-stall trim conditions, this does not imply that such a control feedback would not exist. Concluding our study, we proposed a reformulated sum-of-squares control synthesis based on infinite-horizon backwards-reachability of a target trim condition without the necessity of an ellipsoidal shape. Indeed, a simple linear control feedback could thus be verified to recover the aircraft. We conclude that sum-of-squares programming combined with piecewise polynomial models is a powerful tool for aircraft analysis and verification. 


\section{Appendix}

We provide details of the polynomial aircraft model, the sum-of-squares computations, and the computation of quasi-ellipsoidal surfaces using SOS programming.

\section{A. Details on the polynomial aircraft model}

The low-angle of attack trim condition $\mathbf{x}^{*}$ for gliding descent in Section $\Pi 11$ is given by

$$
V_{\mathrm{A}}^{*}=11.3631 \mathrm{~m} / \mathrm{s}, \quad \gamma_{\mathrm{A}}^{*}=-2.2834^{\circ}, \quad q^{*}=0^{\circ} / \mathrm{s}, \quad \alpha^{*}=3.2240^{\circ}, \quad \eta^{*}=-5^{\circ},
$$

and the deep-stall target trim condition $\mathbf{x}_{1}$ in Section [V] by

$$
V_{\mathrm{A} 1}=8.3131 \mathrm{~m} / \mathrm{s}, \quad \gamma_{\mathrm{A} 1}=-35.5372^{\circ}, \quad q_{1}=0^{\circ} / \mathrm{s}, \quad \alpha_{1}=31.6781^{\circ}, \quad \eta_{1}=-40^{\circ} .
$$

The additional deep-stall conditions are given by

$$
\begin{array}{lllll}
V_{\mathrm{A} 2}=5.0000 \mathrm{~m} / \mathrm{s}, & \gamma_{\mathrm{A} 2}=-57.2958^{\circ}, & q_{2}=0^{\circ} / \mathrm{s}, & \alpha_{2}=29.8081^{\circ}, & \eta_{2}=-38^{\circ} ; \\
V_{\mathrm{A} 3}=18.3333 \mathrm{~m} / \mathrm{s}, & \gamma_{\mathrm{A} 3}=-44.5634^{\circ}, & q_{3}=0^{\circ} / \mathrm{s}, & \alpha_{3}=32.5836^{\circ}, & \eta_{3}=-41^{\circ} ; \\
V_{\mathrm{A} 4}=16.6667 \mathrm{~m} / \mathrm{s}, & \gamma_{\mathrm{A} 4}=-19.0986^{\circ}, & q_{4}=0^{\circ} / \mathrm{s}, & \alpha_{4}=35.1836^{\circ}, & \eta_{4}=-44^{\circ} ; \\
V_{\mathrm{A} 5}=15 \mathrm{~m} / \mathrm{s}, & \gamma_{\mathrm{A} 5}=6.3662^{\circ}, & q_{5}=0^{\circ} / \mathrm{s}, & \alpha_{5}=37.6092^{\circ}, & \eta_{5}=-47^{\circ} ; \\
V_{\mathrm{A} 6}=13.3333 \mathrm{~m} / \mathrm{s}, & \gamma_{\mathrm{A} 6}=31.8310^{\circ}, & q_{6}=0^{\circ} / \mathrm{s}, & \alpha_{6}=39.8574^{\circ}, & \eta_{6}=-50^{\circ} ; \\
V_{\mathrm{A} 7}=11.6667 \mathrm{~m} / \mathrm{s}, & \gamma_{\mathrm{A} 7}=57.2958^{\circ}, & q_{7}=0^{\circ} / \mathrm{s}, & \alpha_{7}=41.9214^{\circ}, & \eta_{7}=-53^{\circ} .
\end{array}
$$

The piecewise model of Eqs. (1)-(4) is not immediately suitable for sum-of-squares analysis. The nonpolynomial sine and cosine functions in $\gamma_{\mathrm{A}}$ and $\alpha$ are therefore approximated by 5th-order and 4th-order Taylor series expansions,

$$
\sin a \approx a-\frac{a^{3}}{3 !}+\frac{a^{5}}{5 !} ; \quad \cos a \approx 1-\frac{a^{2}}{2}+\frac{a^{4}}{4 !}
$$

respectively, which have an error of less than \pm 0.02 for $\left.\gamma_{\mathrm{A}}, \alpha \in\right]-90^{\circ} ;+90^{\circ}\left[\right.$. The inversion of $V_{\mathrm{A}}$ in Eq. (2) is likewise replaced by

$$
a^{-1} \approx a_{0}^{-1}-a_{0}^{-2}\left(a-a_{0}\right)+2 a_{0}^{-3}\left(a-a_{0}\right)^{2}-3 ! a_{0}^{-4}\left(a-a_{0}\right)^{3},
$$


where $a_{0}$ denotes the airspeed in trim. The approximation of the inverse is less accurate than the approximation of the trigonometric functions, however, simulations of the resulting piecewise polynomial equations of motion indicated that the polynomial diverges where the nonlinear model converges, rather than the opposite; that is, analysis of the piecewise polynomial model allows for a conservative stability estimate.

The uncontrolled piecewise polynomial model with the polynomial approximations above is of order 13 . In order to ease the complexity of the SOS computation, we have removed any polynomial term of 6 th order or higher or with coefficients absolutely smaller than $10^{-6}$.

\section{B. Details on the SOS computations}

We provide details about the sum-of-squares computations, polynomial degrees of the problems, and the decision variables involved, respectively. Table 2 details the results of the different SOS computations in Sections III and IV] in terms of the pseudo-radii $\rho^{\diamond}$ of the ellipsoidal shapes $\mathcal{E}_{\rho}$ and degree of reachability $\vartheta^{\diamond}$, respectively, as well as the level sets for invariance and invariance under control, $\lambda^{\diamond}$ and $\lambda^{*}$. The number of involved states is further given as well as the number of repetitions of the (extended) $\mathcal{V}-s$-iteration and the total computation time. Table 3 reports the polynomial degrees of control system, Lyapunov functions, positive terms $l_{a, b}$, ellipsoidal shape functions, and constraints. In addition, Table 4 gives the chosen degrees for the sum-of-squares multipliers in Eqs. (9)- 16 .

\section{Computing quasi-ellipsoids with SOS}

If the Cartesian dimension, that is, the number of variables, or the desired polynomial order of a quasiellipsoid grows, so does the number of independent coefficients. Thus, selecting an ellipsoidal manually becomes increasingly difficult. In order to obtain the asymmetric shapes in Sections III B 2 and III B 3 , we have solved the following sum-of-squares problem given a sequence of points $\mathbf{x}_{1}^{\circ}, \ldots, \mathbf{x}_{k}^{\circ} \in \mathbb{R}^{n}, k \in \mathbb{N}$ finite: find $P \in \mathbb{R}[\mathbf{x}]$ such that $P$ is positive-definite and

$$
P\left(\mathbf{x}_{i}^{\circ}\right)=1
$$

for all $1 \leq i \leq k$. As the degree of $P$ is predefined, the equality constraints in 21 are linear in the decision variables (the coefficients of $P$ ).

The 4th-order, asymmetric shape functions $\tilde{P}_{\text {poly }}$ and $\tilde{P}_{\mathrm{pw}}$ are thus derived to (coefficients are subject to 
Table 2 Results of SOS computations.

(a) Stability analysis (Intel Core i7, $3 \mathrm{GHz}, 16 \mathrm{~GB}$ ).

\begin{tabular}{|c|c|c|c|c|c|c|c|}
\hline Section & Alg. & $n$ & $\rho^{\diamond}$ & $\lambda^{\diamond}$ & $\lambda^{*}$ & Iterations & Time \\
\hline Sec. III A & 1 & 4 & 54.5349 & 1.0513 & 1.0513 & 135 & $3.8295 \mathrm{~h}$ \\
\hline
\end{tabular}

(b) Control synthesis (Intel Core E5, $3 \mathrm{GHz}, 16 \mathrm{~GB}$ ).

\begin{tabular}{|c|c|c|c|c|c|c|c|c|c|}
\hline Section & & Alg. & $n$ & $\rho^{\diamond}$ & $\lambda^{\diamond}$ & $\lambda^{*}$ & Iterations & Time & \\
\hline \multirow{3}{*}{ Sec. IIIB. } & \multirow{3}{*}{$\begin{array}{l}1 \\
1 \\
\end{array}$} & \multirow{3}{*}{1} & \multirow{3}{*}{5} & 39.0320 & 38.7939 & 38.5986 & 109 & 36.4526 & $\mathrm{~h}$ \\
\hline & & & & 251.9531 & 35.3516 & 38.6230 & 70 & 18.4957 & \\
\hline & & & & 295.6543 & 17.7979 & 19.1650 & 73 & 21.1351 & $\mathrm{~h}$ \\
\hline
\end{tabular}

(c) Modified implementation without ellipsoidal shape (Intel Core i7, $3 \mathrm{GHz}, 16 \mathrm{~GB}$ ).

\begin{tabular}{|c|c|c|c|c|c|c|c|c|c|}
\hline \multicolumn{2}{|c|}{ Section } & Alg. & $n$ & $\rho^{\diamond}$ & $\lambda^{\diamond}$ & $\lambda^{*}$ & Iterations & Time & \\
\hline Sec. III A & \multirow[b]{2}{*}{1} & \multirow{2}{*}{13} & 4 & - & 0.2906 & 0.2906 & 21 & 0.4176 & $\mathrm{~h}$ \\
\hline Sec. $\overline{\text { III }} \overline{\bar{B}}$ & & & 5 & - & 0.6965 & 0.6965 & 26 & 5.0432 & $\mathrm{~h}$ \\
\hline
\end{tabular}

(d) Deep-stall recovery (Intel Core i7, $3 \mathrm{GHz}, 16 \mathrm{~GB})$.

\begin{tabular}{cccccccccc}
\multicolumn{2}{c}{ Section } & Alg. & $n$ & $\vartheta^{\diamond}$ & $\lambda^{\diamond}$ & $\lambda^{*}$ & Iterations & Time & \\
\hline Sec. IV & 2 & 2 & 5 & 1.3027 & 57.5684 & 99.9023 & 29 & 4.5832 & $\mathrm{~h}$ \\
\hline
\end{tabular}

Table 3 Polynomial degrees of SOS problems.

\begin{tabular}{|c|c|c|c|c|c|c|c|c|}
\hline \multicolumn{2}{|l|}{ Section } & $\mathbf{f}$ & $\mathbf{K}$ & $\mathbf{f}_{\mathbf{K}}$ & $\mathcal{V}_{1,2}$ & $l_{a, b}$ & $P$ & $p_{\eta}$ \\
\hline Sec. III A & & 5 & 1 & 5 & 4 & 2 & 2 & 2 \\
\hline & 1 & & 1 & & & & & \\
\hline Sec. IIIB & 2 & 5 & 3 & 5 & 4 & 2 & 2 & 2 \\
\hline & 3 & & 3 & & & & & \\
\hline Sec. IV & & 5 & 1 & 5 & 4 & 2 & - & 2 \\
\hline
\end{tabular}

Table 4 Polynomial degrees of SOS multipliers.

\begin{tabular}{|c|c|c|c|c|c|c|c|}
\hline Section & & $s_{i, \lambda}$ & $s_{i, \varphi}$ & $s_{\varphi, i}$ & $s_{-\varphi, i}$ & $s_{i, P}$ & $s_{\eta, \lambda^{\prime}}$ \\
\hline Sec. III A & & 2 & 2 & 2 & 2 & 1 & 1 \\
\hline Sec. IIIB & $\frac{1}{2}$ & 2 & 2 & 2 & 2 & 1 & 1 \\
\hline Sec. IV & & 2 & 2 & 2 & 2 & - & 1 \\
\hline
\end{tabular}


rounding; coefficients $<10^{-3}$ are omitted):

$$
\begin{aligned}
& \tilde{P}_{\text {poly }}=5.37 \times 10^{2} \tilde{V}_{\mathrm{A}}^{4}-2.90 \times 10^{1} \tilde{V}_{\mathrm{A}}^{3} \tilde{\gamma}_{\mathrm{A}}-1.48 \times 10^{-3} \tilde{V}_{\mathrm{A}}^{3} \tilde{q}+5.42 \times 10^{2} \tilde{V}_{\mathrm{A}}^{2} \tilde{\alpha}^{2}+2.41 \times 10^{2} \tilde{V}_{\mathrm{A}}^{2} \tilde{\eta}^{2} \\
& +4.11 \times 10^{2} \tilde{V}_{\mathrm{A}}^{2} \tilde{\gamma}_{\mathrm{A}}^{2}+5.42 \times 10^{2} \tilde{V}_{\mathrm{A}}^{2} \tilde{q}^{2}-2.51 \times 10^{-3} \tilde{V}_{\mathrm{A}} \tilde{\alpha}^{2} \tilde{\eta}-2.84 \times 10^{-3} \tilde{V}_{\mathrm{A}} \tilde{\alpha} \tilde{q}^{2}-2.61 \times 10^{2} \tilde{V}_{\mathrm{A}} \tilde{\gamma}_{\mathrm{A}}^{3} \\
& +1.09 \times 10^{-3} \tilde{V}_{\mathrm{A}} \tilde{\gamma}_{\mathrm{A}} \tilde{q}^{2}+5.00 \times 10^{2} \tilde{\alpha}^{4}-1.33 \times 10^{-3} \tilde{\alpha}^{3} \tilde{\gamma}_{\mathrm{A}}-2.65 \times 10^{-3} \tilde{\alpha}^{3} \tilde{q}+2.41 \times 10^{2} \tilde{\alpha}^{2} \tilde{\eta}^{2} \\
& -1.89 \times 10^{-3} \tilde{\alpha}^{2} \tilde{\eta} \tilde{\gamma}_{\mathrm{A}}+5.42 \times 10^{2} \tilde{\alpha}^{2} \tilde{\gamma}_{\mathrm{A}}^{2}-1.93 \times 10^{-3} \tilde{\alpha}^{2} \tilde{\gamma}_{\mathrm{A}} \tilde{q}+5.42 \times 10^{2} \tilde{\alpha}^{2} \tilde{q}^{2}+2.04 \times 10^{1} \tilde{\eta}^{4} \\
& +2.41 \times 10^{2} \tilde{\eta}^{2} \tilde{\gamma}_{\mathrm{A}}^{2}+2.41 \times 10^{2} \tilde{\eta}^{2} \tilde{q}^{2}+1.35 \times 10^{-3} \tilde{\eta} \tilde{\gamma}_{\mathrm{A}} \tilde{q}^{2}+1.38 \times 10^{-3} \tilde{\eta} \tilde{q}^{3}+1.50 \times 10^{2} \tilde{\gamma}_{\mathrm{A}}^{4} \\
& +5.42 \times 10^{2} \tilde{\gamma}_{\mathrm{A}}^{2} \tilde{q}^{2}-2.46 \times 10^{-3} \tilde{\gamma}_{\mathrm{A}} \tilde{q}^{3}+5.00 \times 10^{2} \tilde{q}^{4}-4.93 \times 10^{-3} \tilde{\alpha}^{2} \tilde{\eta}-2.34 \times 10^{-3} \tilde{\alpha} \tilde{q}^{2} \\
& +1.17 \times 10^{2} \tilde{\eta}^{3}+5.04 \times 10^{2} \tilde{V}_{\mathrm{A}}^{2}-2.24 \times 10^{2} \tilde{V}_{\mathrm{A}} \tilde{\gamma}_{\mathrm{A}}+5.00 \times 10^{2} \tilde{\alpha}^{2}+6.63 \times 10^{-3} \tilde{\alpha} \tilde{q} \\
& +2.23 \times 10^{2} \tilde{\eta}^{2}+2.06 \times 10^{2} \tilde{\gamma}_{\mathrm{A}}^{2}+5.00 \times 10^{2} \tilde{q}^{2} \\
& \tilde{P}_{\mathrm{pw}}=5.41 \times 10^{2} \tilde{V}_{\mathrm{A}}^{4}-2.93 \times 10^{1} \tilde{V}_{\mathrm{A}}^{3} \tilde{\gamma}_{\mathrm{A}}-1.21 \times 10^{-3} \tilde{V}_{\mathrm{A}}^{3} \tilde{q}+5.46 \times 10^{2} \tilde{V}_{\mathrm{A}}^{2} \tilde{\alpha}^{2}+2.07 \times 10^{-3} \tilde{V}_{\mathrm{A}}^{2} \tilde{\alpha} \tilde{q} \\
& +2.43 \times 10^{2} \tilde{V}_{\mathrm{A}}^{2} \tilde{\eta}^{2}-1.44 \times 10^{-3} \tilde{V}_{\mathrm{A}}^{2} \tilde{\eta} \tilde{q}+4.15 \times 10^{2} \tilde{V}_{\mathrm{A}}^{2} \tilde{\gamma}_{\mathrm{A}}^{2}+1.69 \times 10^{-3} \tilde{V}_{\mathrm{A}}^{2} \tilde{\gamma}_{\mathrm{A}} \tilde{q}+5.46 \times 10^{2} \tilde{V}_{\mathrm{A}}^{2} \tilde{q}^{2} \\
& -1.12 \times 10^{-3} \tilde{V}_{\mathrm{A}} \tilde{\alpha}^{2} \tilde{q}-2.02 \times 10^{-3} \tilde{V}_{\mathrm{A}} \tilde{\alpha} \tilde{\eta} \tilde{q}+1.32 \times 10^{-3} \tilde{V}_{\mathrm{A}} \tilde{\alpha} \tilde{\gamma}_{\mathrm{A}}^{2}-2.63 \times 10^{2} \tilde{V}_{\mathrm{A}} \tilde{\gamma}_{\mathrm{A}}^{3}-2.86 \times 10^{-3} \tilde{V}_{\mathrm{A}} \tilde{\gamma}_{\mathrm{A}} \tilde{q}^{2} \\
& -1.33 \times 10^{-3} \tilde{V}_{\mathrm{A}} \tilde{q}^{3}+5.24 \times 10^{2} \tilde{\alpha}^{4}+1.51 \times 10^{1} \tilde{\alpha}^{3} \tilde{\eta}+1.10 \times 10^{-3} \tilde{\alpha}^{3} \tilde{\gamma}_{\mathrm{A}}+1.11 \times 10^{-3} \tilde{\alpha}^{3} \tilde{q} \\
& +1.91 \times 10^{2} \tilde{\alpha}^{2} \tilde{\eta}^{2}+1.05 \times 10^{-3} \tilde{\alpha}^{2} \tilde{\eta} \tilde{\gamma}_{\mathrm{A}}+5.46 \times 10^{2} \tilde{\alpha}^{2} \tilde{\gamma}_{\mathrm{A}}^{2}+4.00 \times 10^{-3} \tilde{\alpha}^{2} \tilde{\gamma}_{\mathrm{A}} \tilde{q}+5.46 \times 10^{2} \tilde{\alpha}^{2} \tilde{q}^{2} \\
& +7.84 \times 10^{1} \tilde{\alpha} \tilde{\eta}^{3}-1.58 \times 10^{-3} \tilde{\alpha} \tilde{\eta} \tilde{q}^{2}-1.32 \times 10^{-3} \tilde{\alpha} \tilde{\gamma}_{\mathrm{A}}^{2} \tilde{q}+1.05 \times 10^{-3} \tilde{\alpha} \tilde{\gamma}_{\mathrm{A}} \tilde{q}^{2}+2.89 \times 10^{1} \tilde{\eta}^{4} \\
& +2.43 \times 10^{2} \tilde{\eta}^{2} \tilde{\gamma}_{\mathrm{A}}^{2}+2.43 \times 10^{2} \tilde{\eta}^{2} \tilde{q}^{2}-2.17 \times 10^{-3} \tilde{\eta} \tilde{q}^{3}+1.51 \times 10^{2} \tilde{\gamma}_{\mathrm{A}}^{4}+5.46 \times 10^{2} \tilde{\gamma}_{\mathrm{A}}^{2} \tilde{q}^{2} \\
& +3.83 \times 10^{-3} \tilde{\gamma}_{\mathrm{A}} \tilde{q}^{3}+5.00 \times 10^{2} \tilde{q}^{4}+1.69 \times 10^{-3} \tilde{V}_{\mathrm{A}} \tilde{\alpha} \tilde{\eta}-1.86 \times 10^{-3} \tilde{V}_{\mathrm{A}} \tilde{\gamma}_{\mathrm{A}} \tilde{q}-1.15 \times 10^{-3} \tilde{V}_{\mathrm{A}} \tilde{q}^{2} \\
& -4.61 \times 10^{1} \tilde{\alpha}^{3}+3.65 \times 10^{1} \tilde{\alpha}^{2} \tilde{\eta}+1.58 \times 10^{-3} \tilde{\alpha}^{2} \tilde{\gamma}_{\mathrm{A}}+2.24 \times 10^{-3} \tilde{\alpha}^{2} \tilde{q}-8.84 \times 10^{1} \tilde{\alpha} \tilde{\eta}^{2} \\
& +1.57 \times 10^{-3} \tilde{\alpha} \tilde{q}^{2}+1.06 \times 10^{2} \tilde{\eta}^{3}+1.07 \times 10^{-3} \tilde{\gamma}_{\mathrm{A}} \tilde{q}^{2}+5.08 \times 10^{2} \tilde{V}_{\mathrm{A}}^{2}-2.27 \times 10^{2} \tilde{V}_{\mathrm{A}} \tilde{\gamma}_{\mathrm{A}} \\
& +1.19 \times 10^{-3} \tilde{V}_{\mathrm{A}} \tilde{q}+5.22 \times 10^{2} \tilde{\alpha}^{2}+2.25 \times 10^{1} \tilde{\alpha} \tilde{\eta}+2.21 \times 10^{2} \tilde{\eta}^{2}+2.05 \times 10^{2} \tilde{\gamma}_{\mathrm{A}}^{2} \\
& +1.06 \times 10^{-3} \tilde{\gamma}_{\mathrm{A}} \tilde{q}+5.00 \times 10^{2} \tilde{q}^{2} ;
\end{aligned}
$$

shape functions are defined in scaled variables.

\section{Funding Sources}

This work was funded by ONERA - The French Aerospace Lab, République Française. 


\section{References}

[1] Philippe, C., "Verification, Validation, and Certification of Aerospace Control Systems," The Impact of Control Technology, edited by T. Samad and A. M. Annaswamy, IEEE Control Systems Society, New York, US-NY, 2011, Chap. 4, pp. 205-206. URL http://www.ieeecss.org/general/impact-control-technology.

[2] Goman, M. G., Zagainov, G. I., and Khramtsovsky, A. V., "Application of bifurcation methods to nonlinear flight dynamics problems," Progress in Aerospace Sciences, Vol. 33, No. 9-10, 1997, pp. 539-586. doi:10.1016/S03760421(97)00001-8.

[3] Gill, S. J., Lowenberg, M. H., Neild, S. A., Krauskopf, B., Puyou, G., and Coetzee, E., "Upset Dynamics of an Airliner Model: A Nonlinear Bifurcation Analysis," Journal of Aircraft, Vol. 50, No. 6, 2013, pp. $1832-1842$. doi:10.2514/1.C032221.

[4] McDonough, K., Kolmanovsky, I., and Atkins, E., "Recoverable sets of initial conditions and their use for aircraft flight planning after a loss of control event," AIAA Guidance, Navigation, and Control Conference, National Harbor, US-MD, 2014. doi:10.2514/6.2014-0786.

[5] McDonough, K., and Kolmanovsky, I., "Fast Computable Recoverable Sets and Their Use for Aircraft Lossof-Control Handling," Journal of Guidance, Control, and Dynamics, Vol. 40, No. 4, 2017, pp. 934-947. doi: 10.2514/G001747.

[6] Lygeros, J., "On reachability and minimum cost optimal control," Automatica, Vol. 40, No. 6, 2004, pp. 917-927. doi:10.1016/j.automatica.2004.01.012.

[7] Nabi, H. N., Lombaerts, T. J. J., Zhang, Y., van Kampen, E., Chu, Q. P., and de Visser, C. C., "Effects of Structural Failure on the Safe Flight Envelope of Aircraft," Journal of Guidance, Control, and Dynamics, Vol. 41, No. 6, 2018, pp. 1257-1275. doi:10.2514/1.G003184.

[8] Genesio, R., Tartaglia, M., and Vicino, A., "On the Estimation of Asymptotic Stability Regions: State of the Art and New Proposals," IEEE Transactions on Automatic Control, Vol. 30, No. 8, 1985, pp. 747-755. doi:10.1109/TAC.1985.1104057.

[9] Tan, W., "Nonlinear Control Analysis and Synthesis using Sum-of-Squares Programming," Phd thesis, University of California, Berkeley, Berkeley, US-CA, 2006.

[10] Seiler, P., and Balas, G. J., "Quasiconvex sum-of-squares programming," 49th IEEE Conference on Decision and Control, Atlanta, US-GA, 2010, pp. 3337-3342. doi:10.1109/CDC.2010.5717672.

[11] Parillo, P. A., "Semidefinite programming relaxations for semialgebraic problems," Mathematical Programming, Series B, Vol. 96, No. 2, 2003, pp. 293-320. doi:10.1007/s10107-003-0387-5. 
[12] Papachristodoulou, A., and Prajna, S., "Robust Stability Analysis of Nonlinear Hybrid Systems," IEEE Transactions on Automatic Control, Vol. 54, No. 5, 2009, pp. 1035-1041. doi:10.1109/TAC.2009.2017155.

[13] Topcu, U., Packard, A., and Seiler, P., "Local stability analysis using simulations and sum-of-squares programming," Automatica, Vol. 44, No. 10, 2008, pp. 2669-2675. doi:10.1016/j.automatica.2008.03.010.

[14] Jarvis-Wloszek, Z., Feeley, R., Tan, W., Sun, K., and Packard, A., "Some Controls Applications of Sum of Squares Programming," Proceedings of the IEEE Conference on Decision and Control, Vol. 5, Maui, US-HI, 2003, pp. 4676-4681. doi:10.1109/CDC.2003.1272309.

[15] Majumdar, A., Ahmadi, A. A., and Tedrake, R., "Control Design Along Trajectories via Sum of Squares Optimization," 2013 IEEE International Conference on Robotics and Automation, Karlsruhe, DE, 2013, pp. 4039-4046. doi:10.1109/ICRA.2013.6631149.

[16] Anderson, J., and Papachristodoulou, A., "Robust nonlinear stability and performance analysis of an F/A-18 aircraft model using sum of squares programming," International Journal of Robust and Nonlinear Control, Vol. 23, 2012, pp. 1099-1114. doi:10.1002/rnc.2928.

[17] Chakraborty, A., Seiler, P., and Balas, G. J., "Nonlinear region of attraction analysis for flight control verification and validation," Control Engineering Practice, Vol. 19, No. 4, 2011, pp. 335-345. doi:10.1016/j.conengprac.2010. 12.001.

[18] Chakraborty, A., Seiler, P., and Balas, G. J., "Susceptibility of F/A-18 Flight Controllers to the Falling-leaf Mode: Nonlinear Analysis," Journal of Guidance, Control, and Dynamics, Vol. 34, No. 2, 2011 , pp. 73-85. doi:10.2514/1.50675.

[19] Tol, H. J., de Visser, C. C., Sun, L. G., van Kampen, E., and Chu, Q. P., "Multivariate Spline-Based Adaptive Control of High-Performance Aircraft with Aerodynamic Uncertainties," Journal of Guidance, Control, and Dynamics, Vol. 39, No. 4, 2016, pp. 781-800. doi:10.2514/1.G001079.

[20] Cunis, T., Burlion, L., and Condomines, J.-P., "Piecewise Polynomial Modeling for Control and Analysis of Aircraft Dynamics beyond Stall," Journal of Guidance, Control, and Dynamics, Vol. 42, No. 4, 2019, pp. 949-957. doi:10.2514/1.G003618.

[21] Cunis, T., Condomines, J.-P., Burlion, L., and la Cour-Harbo, A., "Dynamic Stability Analysis of Aircraft Flight in Deep Stall," Journal of Aircraft, Vol. 57, No. 1, 2020, pp. 143-155. doi:10.2514/1.C035455.

[22] ISO 1151-1, Flight dynamics - Concepts, quantities and symbols - Part 1: Aircraft motion relative to the air, $4^{\text {th }}$ ed., International Organization for Standarization, Genève, CH, 1988.

[23] Chang, B.-C., Kwatny, H. G., Ballouz, E. R., and Hartmann, D. C., "Aircraft Trim Recovery from Highly Nonlinear Upset Conditions," AIAA Guidance, Navigation, and Control Conference, San Diego, US-CA, 2016. doi:10.2514/6.2016-0880. 
[24] Kwatny, H. G., Dongmo, J.-E. T., Chang, B.-C., Bajpai, G., Yasar, M., and Belcastro, C., "Nonlinear Analysis of Aircraft Loss of Control," Journal of Guidance, Control, and Dynamics, Vol. 36, No. 1, 2013, pp. 149-162. doi:10.2514/1.56948.

[25] Cunis, T., and la Cour-Harbo, A., "Piecewise Polynomial Model of the Aerodynamic Coefficients of the Cumulus One Unmanned Aircraft," Tech. Rep. hal-02280789, Sky-Watch A/S, Støvring, DK, 2019. URL https:// archives-ouvertes.fr/hal-02280789

[26] Yin, H., Packard, A., Arcak, M., and Seiler, P., "Finite horizon backward reachability analysis and control synthesis for uncertain nonlinear systems," Proceedings of the American Control Conference, Philadelphia, US-PA, 2019, pp. 5020-5026. doi:10.23919/ACC.2019.8814444.

[27] Zheng, X., She, Z., Lu, J., and Li, M., "Computing multiple Lyapunov-like functions for inner estimates of domains of attraction of switched hybrid systems," International Journal of Robust and Nonlinear Control, Vol. 28, No. 17, 2018, pp. 5191-5212. doi:10.1002/rnc.4280.

[28] Cunis, T., "Toolbox for region-of-attraction estimation of piecewise-defined polynomial systems using sumof-squares prgramming," , 2019. URL https://github.com/pwpfit/pwroaest, revision 27716d2, accessed $02 / 21 / 2020$.

[29] Khodadadi, L., Samadi, B., and Khaloozadeh, H., "Estimation of region of attraction for polynomial nonlinear systems: A numerical method," ISA Transactions, Vol. 53, No. 1, 2014, pp. 25-32. doi:10.1016/j.isatra.2013.08.005.

[30] Jones, M., Mohammadi, H., and Peet, M. M., "Estimating the region of attraction using polynomial optimization: A converse Lyapunov result," 56th IEEE Conference on Decision and Control, Melbourne, AU, 2017, pp. 1796-1802. doi:10.1109/CDC.2017.8263908.

[31] Chesi, G., "On the Complexity of SOS Programming and Applications in Control Systems," Asian Journal of Control, Vol. 20, No. 5, 2018, pp. 2005-2013. doi:10.1002/asjc.1684.

[32] Cunis, T., Condomines, J.-P., and Burlion, L., "Local stability analysis for large polynomial spline systems," Automatica, Vol. 113, 2020. doi:10.1016/j.automatica.2019.108773. 